\title{
High-Frequency Vibration Energy Harvesting from Impulsive Excitation Utilizing Intentional Dynamic Instability Caused by Strong Nonlinearity
}

\author{
Kevin Remick ${ }^{\mathrm{a}}$, D. Dane Quinn ${ }^{\mathrm{b}}$, D. Michael McFarland ${ }^{\mathrm{c}}$, Lawrence \\ Bergman $^{\mathrm{c}}$, Alexander Vakakis ${ }^{\mathrm{a}}$ \\ ${ }^{a}$ Department of Mechanical Science and Engineering, University of Illinois at \\ Urbana-Champaign, 1206 W. Green St., Urbana, IL 61801, USA \\ ${ }^{b}$ Department of Mechanical Engineering, University of Akron, Akron, OH 44325, USA \\ ${ }^{c}$ Department of Aerospace Engineering, University of Illinois at Urbana-Champaign, 104 \\ S. Wright Street, Urbana, IL 61801, USA
}

\begin{abstract}
The authors investigate a vibration-based energy harvesting system utilizing essential (nonlinearizable) nonlinearities and electromagnetic coupling elements. The system consists of a grounded, weakly damped linear oscillator (primary system) subjected to a single impulsive load. This primary system is coupled to a lightweight, damped oscillating attachment (denoted as nonlinear energy sink, NES) via a neodymium magnet and an inductance coil, and a piano wire, which generates an essential geometric cubic stiffness nonlinearity. Under impulsive input, the transient damped dynamics of this system exhibit transient resonance captures (TRCs) causing intentional large-amplitude and high-frequency instabilities in the response of the NES. These TRCs result in strong energy transfer from the directly excited primary system to the light-weight attachment. The energy is harvested by the electromagnetic elements in the coupling and, in the present case, dissipated in a resistive element in the electrical circuit. The primary goal of this work is to numerically, analytically, and experimentally demonstrate the efficacy of employing this type of intentional high-frequency dynamic instability to achieve enhanced vibration energy harvesting under impulsive excitation.
\end{abstract}

Keywords: Strong nonlinearity, Electromagnetic, Energy harvesting, Impulsive excitation 


\section{Introduction}

Typical vibration energy harvesting systems are based on linear or weakly nonlinear tuned mass dampers (TMD), which consist of an oscillating primary mass coupled to a secondary light-weight absorbing mass. With the primary mass subject to harmonic excitation, these linear systems can be specifically tuned to efficiently harvest energy. This is accomplished by matching the natural frequency of the primary and secondary systems to the external forcing frequency $[1,2]$. Furthermore, proper tuning of the electrical circuit parameters optimizes the electromechanical conversion process, providing for increased harvesting efficiency $[3,4,5,6,7]$. Energy transfer from the primary system to the linear harvester increases for larger primary excitation amplitude and weaker system damping [8]. Low system damping results in a sharp resonant peak, which is indicative of the narrowband operation of these linear harvesting systems. Harvesting efficiency decreases significantly from excitations with time-varying frequency or from frequencies that vary slightly from the tuned resonance frequency of the mechanical system. Optimizing strategies for the former class of excitations were formulated theoretically in [9] for a linear single-degree-of-freedom harvester.

Nonlinear energy harvesting systems have been designed to accommodate frequency variation and frequency mistuning [10, 11, 12]. Mann and Sims [11] investigated magnetic restoring force spacing, which allowed for tunable resonance of an oscillator to input conditions. The authors also discovered experimentally that damping plays a vital role in the ability of the harvester to engage in high-energy responses. Ma and Zhang [13, 14] utilized a pendulum system with a potential well to increase energy harvesting efficiency when the response escapes the well. Modulating forcing frequency and tunable damping were investigated as a means to tune resonance of the system for increased harvesting efficiency. The authors concluded that an active element in the system to keep the oscillator operating outside of the well would greatly benefit the harvesting efficiency. Erturk et al. [15] showed superior harvesting performance for an inverted cantilever beam under buckling conditions and broadband excitation. The authors used piezo-magneto-elastic effects to enhance harvesting compared to systems without these effects.

Incorporating nonlinear mechanical attachments into linear primary systems has been explored in the literature as a means to broaden frequency response range and provide large-amplitude solutions [16, 17, 18]. These

mechanical attachments use strong cubic stiffness nonlinearities to achieve 
this behavior. The literature describes this class of strong nonlinearity as essential (nonlinearizable) nonlinearity. Kremer and Liu [16] use a magnetic restoring force to provide the cubic term in the coupling; however, the authors must also have a linear term in the coupling due to the experimental configuration. Hu et al. and Andersen et al. [17, 18] use geometric effects to provide the cubic term and minimize linear effects in the coupling. These nonlinear systems exhibit the phenomenon of targeted energy transfer (TET) when subject to proper conditions. Targeted energy transfer describes the passive, nearly irreversible transfer of significant energy from the primary system to the nonlinear attachment $[19,20,21]$. Complex dynamics result in these systems due to the capability of the essentially nonlinear attachment to engage in resonance captures with modes of the linear structure [22]. These modes span an extensive frequency and energy range. High co-dimensional bifurcations in the dynamics result in chaotic motions and dynamic instabilities, leading to large relative displacements or high-frequency responses and, thus, good energy harvesting performance. The bifurcations arise from highly degenerate eigenstructures in the underlying Hamiltonian system dynamics, which possess complex conjugate, imaginary, and multiple zero eigenvalues.

Highly degenerate systems associated with geometric stiffness [18] and damping [23] nonlinearities have exhibited these large-amplitude nonlinear instabilities. As the nonlinear attachment engages in a resonance capture with a linear oscillator mode, the instability manifests as a buildup or "burst" in the response of the nonlinear attachment. The authors in [23, 24] describe another dynamical phenomenon that occurs in the neighborhood of the $\mathrm{im}$ pulsive orbit manifold (IOM) of the underlying Hamiltonian system. The lightly damped dynamics of the two coupled oscillators with essential cubic stiffness nonlinearity transitions into a state of sustained nonlinear resonance scattering along the IOM, which consists of a countable infinity of periodic orbits and an uncountable infinity of quasi-periodic orbits. These orbits extend over broad frequency and energy ranges [25] and arise when impulsive forces are applied to the linear oscillator with the system initially at rest. This transition manifests as a nonlinear beating phenomenon. The authors in [23] predict analytically the presence of these transitions in the neighborhood of the low-frequency branch of the IOM and then proceed to prove its existence experimentally for a system with weak viscous damping. The authors in [26] expand upon the previous work to predict the presence of these transitions in the neighborhood of the high-frequency branch of the IOM and again prove the existence experimentally. Remick et al. [27] theoretically explore the ap- 
plication of this high-frequency dynamic instability in an energy harvesting system utilizing piezoelectric coupling elements. The authors showed that the system can be placed into a state of sustained, high-frequency dynamic instability under impulsive loading conditions, which results in superior energy harvesting performance. The authors also theoretically examine the performance of this system when subjected to stochastic impulsive excitation, in which the inter-arrival time and impulse intensity are varied. Several performance measures are presented for identifying cases in which high-frequency dynamic transitions are possible under these stochastic resonance conditions. The authors conclude by identifying baseline minimum performance excitation conditions and optimal performance conditions, which are strongly dependent upon the relative phase states of the two oscillators.

The present work seeks to expand upon the aforementioned results analytically and experimentally for a system of two coupled oscillators with essential cubic stiffness nonlinearity and electromagnetic coupling. Cubic stiffness nonlinearity is produced by means of simple geometric and kinematic effects, which is readily and accurately characterizable. Electromagnetic harvesting elements are used to allow for a larger and more easily tunable transduction factor compared to piezoelectric coupling elements, which is better for this application for system optimization and energy generation. It is demonstrated that the high-frequency instability can be experimentally obtained for a system with moderate viscous damping subject to a single impulsive load, and that this high-frequency instability corresponds to good energy harvesting performance. The analysis seeks to further identify the system behavior over a range of parameter values. This system is explored for purely deterministic excitation conditions, leaving stochastic excitation for future work. Concluding remarks suggest expanding this work to the case of repeated impulsive excitations, which will be the subject of a companion paper. In terms of applications, here the harvester as well as the structure to which it is attached is modeled. The force is applied to the structure and the resulting motion transfers to the harvester. The massive linear oscillator is the primary system, in which the grounding elements simply provide the first mode of the structure. With this understanding, the applications center around any system that is impulsively excited, such as walking or running. 


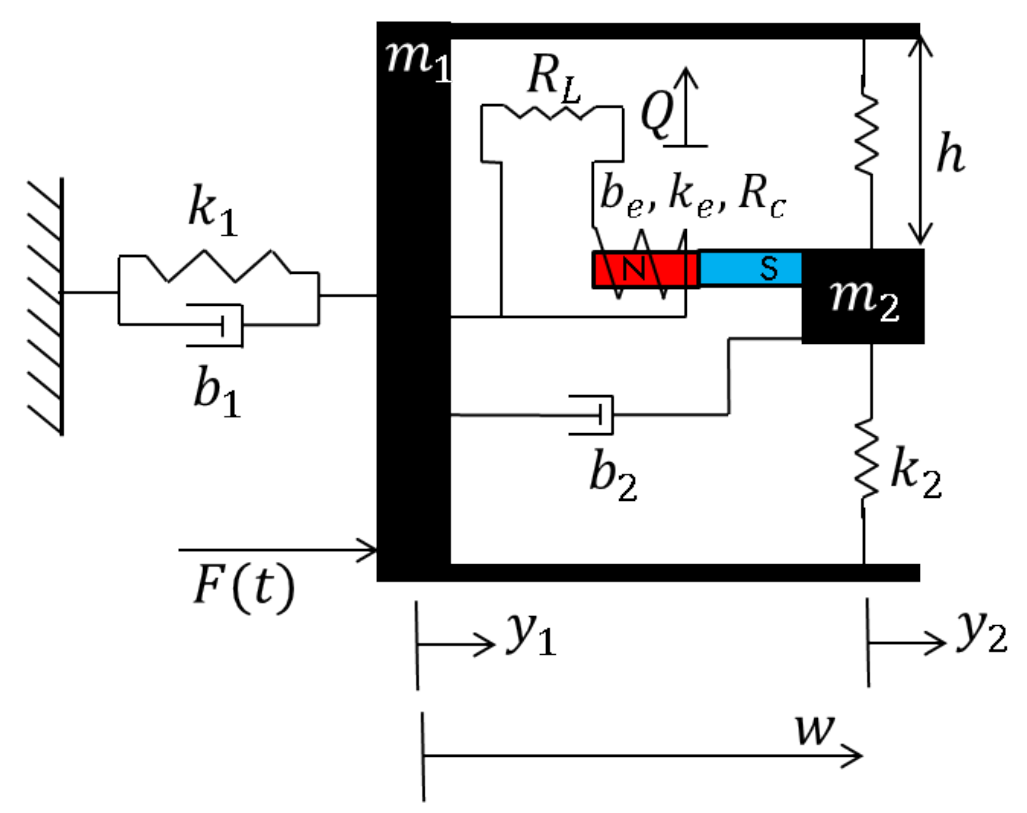

Figure 1: Configuration of the two coupled oscillators with essential geometric nonlinearity and electromagnetic elements. The primary linear oscillator is denoted with 1 subtext while the secondary nonlinear oscillator is denoted with 2 subtext. The two oscillators are coupled via linear stiffness elements, which exhibit strong cubic nonlinear characteristics when deformed in the transverse direction. The two oscillators are also coupled via electromagnetic elements, which are the energy harvesting elements of choice for this study. A simple resistive circuit is used to gage performance. A more in-depth description of the components are presented in the following text. 


\section{System Modeling}

We consider a nonlinear energy harvesting system (cf. Fig. 1) composed of a linear, lightly damped oscillator (primary system) coupled to a lightweight, nonlinear, moderately damped oscillator (nonlinear energy sink, NES) via a permanent magnet, inductance coil, and piano wire. The piano wire generates an essential cubic stiffness nonlinearity through geometric and kinematic nonlinearities that arise due to transverse deformations of the linear springs. The permanent magnet and inductance coil produce a linear, electromechanical damping term in the coupling, which is in addition to linear viscous mechanical damping inherent in the system. A perfectly cylindrical inductance coil and magnet are assumed for the model and resulting analysis. The total coupling force in the direction of oscillation can be expressed as

$$
F_{c}=F_{d}+F_{s}+F_{e}=b_{2} \dot{w}+\frac{k_{2}}{h^{2}} w^{3}+b_{e} \dot{w},
$$

where $b_{2}$ is the linear viscous damping coefficient, $k_{2}$ is the axial stiffness of the piano wire, $h$ is the half-length of the wire, and $b_{e}$ is the electromechanically induced damping coefficient. The absolute displacements of the primary system and NES are denoted by $y_{1}$ and $y_{2}$, respectively. The relative displacement $w$ is defined as $y_{2}-y_{1}$, with time-derivative $\dot{w}$, where $(\cdot) \equiv d / d t$. A force $F(t)$ is applied in the direction of oscillation to the primary system, which is grounded via a linear spring of stiffness $k_{1}$ and linear viscous damping coefficient $b_{1}$. The masses of the primary system and NES are denoted as $m_{1}$ and $m_{2}$, respectively.

The electromechanically induced damping is well-defined by Kremer and Liu [16] and Beeby and O'Donnell [28]. Faraday's law describes the electromotive force $(\mathrm{V})$ induced by a circuit as the time rate of change of the magnetic flux linkage or

$$
\mathrm{V}=-\frac{d \phi}{d t}
$$

where $\phi$ is the magnetic flux linkage. For an $N$-turn coil, the induced electromotive force is defined as a function of the total flux linkage $\Phi$ as

$$
\mathrm{V}=-\frac{d \Phi}{d t}=-N \frac{d \phi}{d t}
$$

The total flux linkage for an $N$-turn coil is evaluated as the combination of 
flux linkages for individual turns, i.e.

$$
\Phi=\sum_{i=1}^{N} \int_{A_{i}} B\left(A_{i}\right) \cdot d A
$$

where $B$ denotes the magnetic field flux density for a given $i^{\text {th }}$ turn area $A_{i}$. The area of the effective magnetic field depends on the magnet's position with respect to the coil $w$ (axial dependence) and the size of the coil in terms of the outer radius $r_{o}$ and inner radius $r_{i}$ (radial dependence). Lenz's law describes the electromagnetic force $F_{e}$ as a function of the current in the coil $\dot{Q}$ and the transduction factor $k_{e}$, or

$$
F_{e}=k_{e} \dot{Q}
$$

The transduction factor is a function of the magnetic field flux density and coil size, thus describing the strength of the electromechanical coupling. The transduction factor can be defined as the change in total flux linkage with position,

$$
k_{e}=\frac{d \Phi}{d w} .
$$

Substituting the expression for the transduction factor in (6) into the expression for Faraday's law in (3), the electomotive force can be described as

$$
\mathrm{V}=k_{e} \dot{w} .
$$

Assuming a uniform flux density over the area of the coil and substituting the expression (4) into (6), the transduction factor can be reduced to the simple expression

$$
k_{e}=\frac{L_{w} h_{c}\left(r_{o}-r_{i}\right)}{A_{c}} B
$$

where $L_{w}$ is the length of the wire in the coil, $h_{c}$ is the axial length of the coil, and $A_{c}$ is the cross-sectional area of the coil, or $A_{c}=\pi\left(r_{o}^{2}-r_{i}^{2}\right)$.

As seen in Fig. 1, power is extracted from the mechanical system by connecting the coil to a fixed load resistance $R_{L}$. The current is able to flow through the coil and load resistance, creating its own magnetic field in opposition to the field created by the permanent magnet. The coil inherently possesses its own resistance $R_{c}$ and inductance $L_{c}$ parameters. The electromagnetic force described with Eqs. (1) and (6) is produced from the 
interaction of the field from the induced current and the field from the permanent magnet, allowing the conversion of mechanical energy into electrical energy. The current in the circuit due to the electromotive force can be described by applying Kirchoff's law to the system, i.e.

$$
\mathrm{V}=L_{c} \ddot{Q}+\left(R_{c}+R_{L}\right) \dot{Q} .
$$

Thus Eq. (9) can be combined with Eq. (7) to express the current in the system as

$$
\dot{Q}=\frac{k_{e}}{\left(R_{c}+R_{L}\right)} \dot{w}-\frac{L_{c}}{\left(R_{c}+R_{L}\right)} \ddot{Q} .
$$

Wheeler in [29] provides an estimate for the coil inductance as

$$
L_{c}=\frac{\left(7.875 \times 10^{-6}\right)\left(r_{o}+r_{i}\right)^{2} N}{13 r_{o}-7 r_{i}+9 h_{c}},
$$

and the maximum coil impedance $Z_{L}$ can be estimated as

$$
Z_{L}=2 \pi f_{\max } L_{c}
$$

where $f_{\max }$ is the maximum driving frequency (in $\mathrm{Hz}$ ) imposed by the mechanical system. As will be shown later, the experimental coil inductance and resulting coil impedance are negligible relative to the load resistance used. Therefore the coil impedance is neglected in further analysis for simplification. Using this assumption, Eq. (10) reduces to

$$
\dot{Q}=\frac{k_{e}}{\left(R_{c}+R_{L}\right)} \dot{w}=\frac{b_{e}}{k_{e}} \dot{w}
$$

where the electromagnetic damping $b_{e}$ is expressed as

$$
b_{e}=\frac{k_{e}^{2}}{R_{c}+R_{L}} .
$$

The nonlinear equations of motion governing the dynamics of the harvesting device can be obtained by summing forces in the direction of oscillation and expanding by means of a Taylor series for the nonlinear terms. The three governing equations are

$$
\begin{gathered}
m_{1} \ddot{y}_{1}+b_{1} \dot{y}_{1}+k_{1} y_{1}-\left(b_{e}+b_{2}\right) \dot{w}-\frac{k_{2}}{h^{2}} w^{3}=F(t), \\
m_{2}\left(\ddot{w}+\ddot{y}_{1}\right)+\left(b_{e}+b_{2}\right) \dot{w}+\frac{k_{2}}{h^{2}} w^{3}=0 \\
\dot{Q}-\frac{b_{e}}{k_{e}} \dot{w}=0 .
\end{gathered}
$$


As seen in (15), the essential cubic stiffness nonlinearity appears in the coupling stiffness.

The preliminary work in [26] theoretically and experimentally demonstrated the ability of introducing high-frequency dynamic instability in the same system but with the electric circuit removed. That work showed that the transient damped dynamics of the system tracks the high-frequency IOM in the frequency-energy plane when the primary system is subject to specific impulse excitation magnitude. Bursts in the response of the NES arise at bifurcation points along damped transitions in the neighborhood of the IOM, resulting in strong energy transfers from the directly excited primary system to the NES. This feature was exploited theoretically in [27] to produce superior energy harvesting performance for the same system with piezoelectric coupling elements and a simple circuit. The principal aim of this study is to show experimentally that high-frequency dynamic instabilities in the response of (15) exist and can provide an effective mechanism for vibration energy harvesting.

The dynamics of the system (15) are explored by introducing nondimensionalized parameters. This is carried out by scaling the time, displacement, and charge variables as $t=c_{t} \tau, y_{1}=c_{x} x, w=c_{x} u$, and $Q=c_{q} q$, respectively. The normalization coefficients are chosen in a manner that normalizes the linear and nonlinear stiffness parameters, as well as one of the circuit parameters. This scaling allows for computational study of the effects of system damping and electromechanical coupling on the performance of the harvester. The normalization coefficients are

$$
c_{t}=\sqrt{\frac{m_{1}}{k_{1}}}, \quad c_{x}=h \sqrt{\frac{k_{1} m_{2}}{k_{2} m_{1}}}, \quad c_{q}=\frac{h k_{1} m_{2}}{k_{e} m_{1}} \sqrt{\frac{m_{2}}{k_{2}}} .
$$

This analysis yields a simplified set of nondimensional equations,

$$
\begin{gathered}
x^{\prime \prime}+\lambda x^{\prime}+x-\mu\left[(\beta+\zeta) u^{\prime}+u^{3}\right]=\gamma f(\tau), \\
u^{\prime \prime}+x^{\prime \prime}+(\beta+\zeta) u^{\prime}+u^{3}=0, \\
q^{\prime}-\beta u^{\prime}=0,
\end{gathered}
$$

where $\gamma f(\tau) \equiv F(t(\tau)),(\cdot)^{\prime} \equiv d / d \tau$, and the nondimensional parameters are 
defined as

$$
\begin{aligned}
& \mu=\frac{m_{2}}{m_{1}}, \quad \lambda=\frac{b_{1}}{\sqrt{m_{1} k_{1}}}, \quad \zeta=\frac{b_{2}}{m_{2}} \sqrt{\frac{m_{1}}{k_{1}}}, \\
& \beta=\frac{b_{e}}{m_{2}} \sqrt{\frac{m_{1}}{k_{1}}}, \quad \gamma=\frac{1}{h k_{1}} \sqrt{\frac{k_{2} m_{1}}{k_{1} m_{2}}}
\end{aligned}
$$

Within these nonlinear, nondimensional equations of motion, the mass ratio between the NES and the primary system is defined by $\mu, \lambda$ defines the linear viscous damping in the primary system, $\zeta$ represents the linear viscous damping in the coupling, $\beta$ describes the electromechanical coupling for the system, and $\gamma$ defines the force magnitude.

A single impulsive excitation scenario is considered in this work. The harvesting system (17) is initially at rest at $\tau=0-$, in which the 0 - denotes the moment just prior to instantaneous zero. A single impulse $\gamma f(\tau)=\tilde{I}_{0} \delta(\tau)$ is then applied to the system at $\tau=0$. Here, we denote $\tilde{I}_{0}=I_{0} \cdot c_{t} / c_{x}$ in which $I_{0}$ is the dimensional initial velocity of the primary system directly following the applied impulse. Experimentally, $I_{0}$ is the maximum velocity that the primary system attains following the applied impulse. Using this assumption of an instantaneously applied force, the equations of motion (17) are complemented by initial conditions

$$
\begin{array}{ll}
x(0+)=0, & x^{\prime}(0+)=\tilde{I}_{0}, \\
u(0+)=0, & u^{\prime}(0+)=-\tilde{I}_{0}, \\
q(0+)=0 &
\end{array}
$$

and $\gamma$ is effectively set equal to zero so that no additional forcing is applied to the system. For further clarification, the instantaneously applied force is expressed as a velocity initial condition. Experimentally, the forcing takes the shape of a half-sine pulse with a specific period, which will be explored in later sections. Recall that $u^{\prime}(\tau)$ describes the nondimensional relative velocity between the oscillators. The initial condition for $u^{\prime}(\tau)$ must be defined as above so that the initial velocity of the NES is zero.

Energy and power expressions can be defined to describe the performance of the energy harvesting system (15) in later sections. The instantaneous power generated by the harvesting device in units of Watts for an impulse magnitude $I_{0}$ can be expressed, in this case, as a measure of the energy 
dissipated across the resistive element in the coupled circuit,

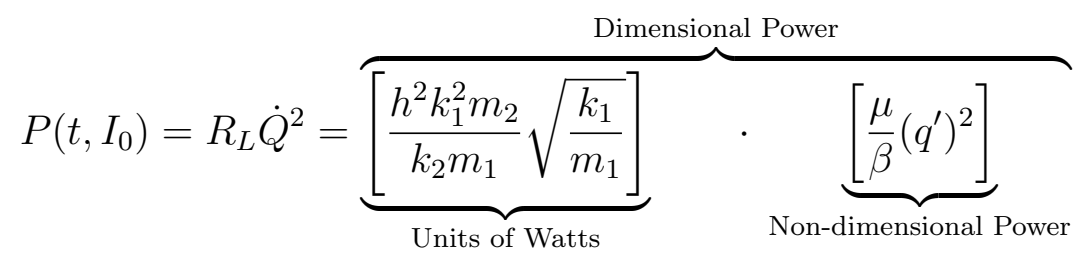

The total energy harvested by the system in units of Joules from $t=0$ to some time $t=t_{f}$ following an impulse of magnitude $I_{0}$ can be expressed as a time integral of the nondimensional power with proper scaling, i.e.

$$
E_{h}\left(t_{f}, I_{0}\right)=\overbrace{\underbrace{\left[\frac{h^{2} k_{1}^{2} m_{2}}{k_{2} m_{1}}\right]}_{\text {Units of Joules }} \cdot \underbrace{\left[\int_{0}^{\tau_{f}} \frac{\mu}{\beta}\left(q^{\prime}(\tau)\right)^{2} d \tau\right]}_{\text {Non-dimensional Energy Harvested }}}^{\text {Dimensional Energy Harvested }}
$$

The energy harvesting efficiency $\eta_{h}$ is expressed in this work as the total energy harvested from time $t=0$ to $t=t_{f}$ after an impulse of magnitude $I_{0}$ normalized by the energy in the system at $t=0+$, or the kinetic energy input into the primary system, i.e.

$$
\eta_{h}\left(t_{f}, I_{0}\right)=\frac{E_{h}\left(t_{f}, I_{0}\right)}{\frac{1}{2} m_{1}\left(I_{0}\right)^{2}} \times 100 \%
$$

As mentioned above, the principal goal of this work is to experimentally demonstrate that effective energy harvesting can be achieved by inducing high-frequency instability of the NES. The dynamics of the underlying Hamiltonian system are discussed in the next section for the experimental apparatus and its inherent parameters. This is necessary to study the nonlinear dynamical mechanism governing the instability and promoting good energy harvesting performance.

\section{Underlying Hamiltonian Dynamics}

The dynamics of the underlying Hamiltonian system derived from (15) can be depicted in a frequency-energy plot (FEP), which is thoroughly explained in $[23,26,27,30]$. The underlying Hamiltonian system is obtained by removing the damping, electrical, and forcing terms from (15), by setting $b_{1}=b_{2}=b_{e}=F(t)=0$. The frequency-energy plane provides a visual 
method by which branches of periodic and quasi-periodic orbits of the underlying Hamiltonian system can be seen for varying energy levels. Transitions between various branches of the FEP are realized in the transient response of the system (15) due to the nonconservative terms in the original equations of motion. Wavelet spectral analysis can be performed by taking the Morlet wavelet transform of a time-history response of the system (15). The wavelet transform is a valuable dynamic time-frequency analysis tool, which is more beneficial than the stationary signal analysis inherent to the fast Fourier transform when considering time series of nonlinear systems whose frequency content may vary with time (and energy level). The resulting wavelet spectra can then be superimposed on the Hamiltonian FEP or plotted versus time to reveal the nonlinear transitions between branches in the transient response. This methodology is based on the assumption that the conservative elements of the Hamiltonian system govern which branch is "visited" by the system for a given energy input, and the nonconservative elements govern the transitions between branches [30]. For clarification, weak damping in the system provides purely parasitic dynamical effects, i.e. damping doesn't introduce any new dynamics into the underlying Hamiltonian system.

The FEP for the underlying Hamiltonian system is shown in Fig. 2 for the experimental parameters $\left(m_{1}, k_{1}, m_{2}, k_{2}\right)$ identified in Table 2 from Section 6. The horizontal dotted line at approximately $8.42 \mathrm{~Hz}$ corresponds to the natural frequency of the primary system. Two global backbone branches, denoted $S 11 \pm$, are then defined over broad frequency and energy ranges. These branches correspond to in-phase and out-of-phase periodic orbits in 1:1 resonance, respectively. These are periodic orbits in which the primary system and the NES oscillate at the same fundamental frequency. The FEP in Fig. 2 also contains two subharmonic tongues, denoted $S 13$ and $S 15$, extending nearly horizontally from the $S 11+$ backbone. These tongues correspond to 1:3 and 1:5 internal resonances, respectively, between the NES and the primary system. These subharmonic internal resonances indicate slower oscillations (lower frequencies) of the NES than the primary system. Conversely, superharmonic internal resonances also exist for this system, although they are not depicted in the FEP presented in Fig. 2. These superharmonic tongues would extend horizontally from the $S 11-$ backbone, indicating resonances in which the NES oscillates faster (at higher frequencies) than the primary system. As discussed in [23,30], a countable infinity of periodic orbits exist in the underlying Hamiltonian system. These occur in pairs of Snm in-phase or out-of-phase subharmonic and superharmonic 


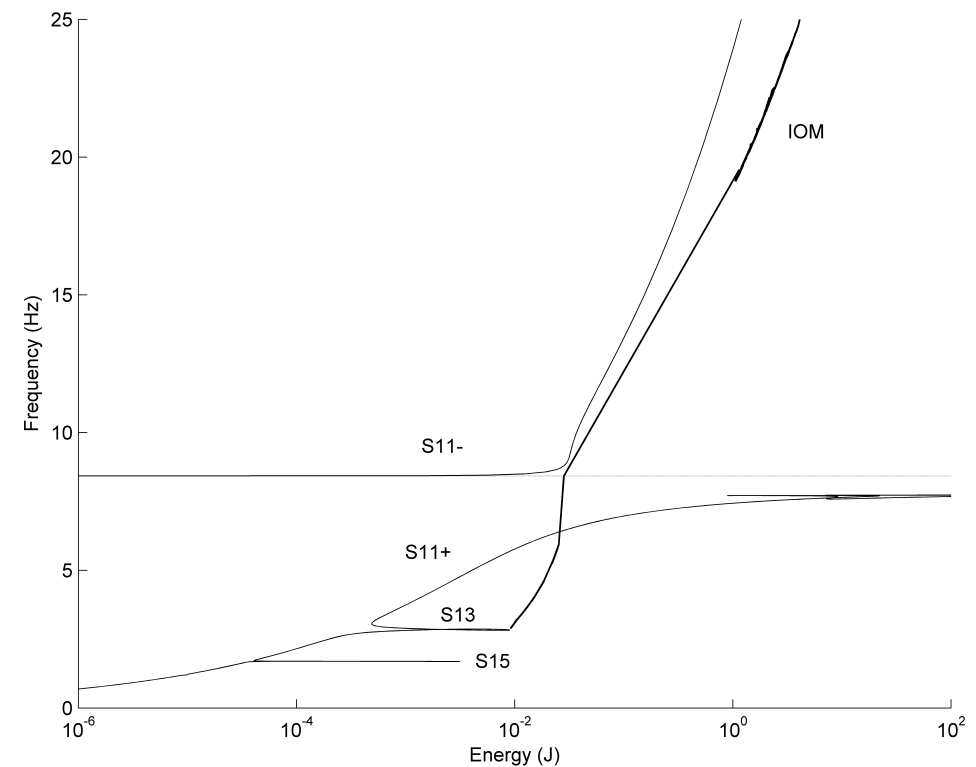

Figure 2: Frequency-energy plot of the underlying Hamiltonian system (15) derived from experimental parameters presented in Table 2 . 
tongues (n:m internal resonances) that exist over finite energy ranges.

The impulsive orbits manifold contains another class of solutions in the FEP. These solutions comprise a countable infinity of periodic orbits and an uncountable infinity of quasi-periodic orbits of the Hamiltonian system. These impulsive orbits correspond to impulsive excitation of the primary system according to initial conditions (19). The previous work in $[23,26,27]$ indicates that orbits of the lightly damped system in the neighborhood of the high-frequency IOM result in strong energy transfers from the primary system to the nonlinear attachment, which is beneficial for harvesting energy from the NES. These strong energy transfers take the form of "transient bursts" (instabilities) in the response of the nonlinear attachment, which arise at bifurcation points along damped transitions near the IOM. These bursts have been described in some works [31, 32] as resembling self-excited resonances.

The next section initiates the computational study of the damped dynamics of system (17) by performing numerical simulations for a single impulse of various magnitudes applied to the primary system. The effect of changing the electromechanical coupling and circuit load resistance will be explored, as well as the determination of physical parameters for the experimental system. Wavelet analysis will be performed on the transient response of the system and then compared to the energy harvesting efficiency defined in (22) to show that these high-frequency transitions can lead to effective energy harvesting.

\section{Numerical Study and System Identification}

The computational study of system (17) is initiated by considering a single impulsive input to the primary system according to (19) and studying the resulting damped transitions via wavelet analysis, as described in the previous section. The numerical simulations carried out in this section are performed with several parameters resulting from an experimental apparatus designed and developed in previous works $[23,26,27]$ by the authors. The experimental apparatus used in this study can be seen in Fig. 12 and is described in more detail in Section 6.

A system identification was performed initially for the mechanical parameter values governing the experimental apparatus so that the computational study could begin with these as a baseline. Linear modal analysis was performed on the primary system alone to extract the parameter values $b_{1}$ 
and $k_{1}$. Nonlinear system identification utilizing the restoring force surface method $[33,34]$ was performed on the attachment alone to extract the parameter values $b_{2}$ and $k_{2}$. The primary system and NES masses were weighed to determine $m_{1}$ and $m_{2}$, respectively, and the piano wire half-span length was measured to determine $h$. These seven mechanical parameter values are presented in Table 2.

As derived in (18), the resulting nondimensional mechanical system parameters can be determined from the identified physical parameter values. The nondimensional system parameters are presented in Table 1. As seen from (18), the mass ratio $\mu$ and damping terms $\lambda$ and $\zeta$ correspond to only the mechanical parameters, leaving the electromechanical coupling coefficient $\beta$ as the only unknown design parameter for the system (17). This electromechanical coupling coefficient is primarily dependent upon the transduction factor $k_{e}$, which is determined by the coil construction and permanent magnet selection. As described in [3, 10], optimized energy harvesting efficiency can be achieved with proper tuning of the electrical circuit parameters. While system optimization is not specifically the goal in this study, parameter optimization can be easily explored with the simplified nondimensional system (17).

The total nondimensional energy harvested from (21) and the energy harvesting efficiency (22) were considered when determining system performance for a range of electromechanical coupling and impulse magnitude values. Appropriate parameter ranges were established according to physical limitations of the experimental apparatus, and then expanded to include other possible outcomes for future apparatus optimization. Contour plots were developed for an electromechanical coupling range $\beta=[0.01,1.00]$ with step $\Delta \beta=0.01$ and impulse magnitude range $\tilde{I}_{0}=[0.05,10]$ with step $\Delta \tilde{I}_{0}=0.05$. The system (17) was numerically integrated with initial conditions (19) for each $\left(\beta, \tilde{I}_{0}\right)$ parameter pair for a given time period $\tau_{f}$. The resulting time series was post-processed to produce performance contours based on energy measures (21) and (22).

The system is integrated for a simulation time of $\tau_{f}=110$, which corresponds physically to approximately $t_{f}=2.0$ seconds. This is a long enough duration to allow the system dynamics to damp out completely while still being short enough for comparing all excitation scenarios. Contour plots for performance measures described in (21) and (22) are depicted in Fig. 3. As indicated by Fig. 3a, the total energy harvested is strongly dependent upon the energy level at which the system operates, or the excitation magnitude, 


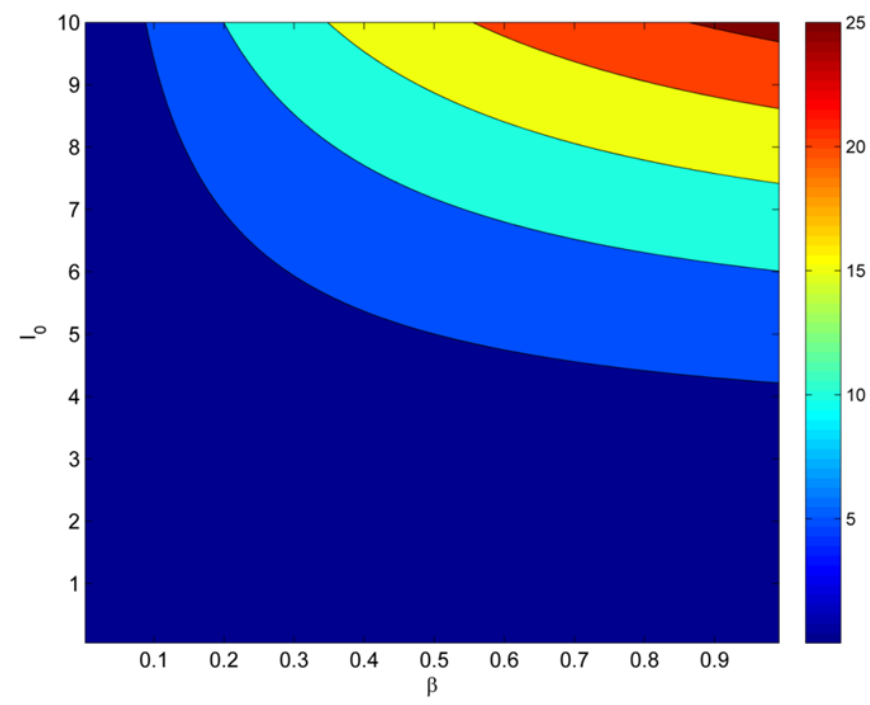

(a)

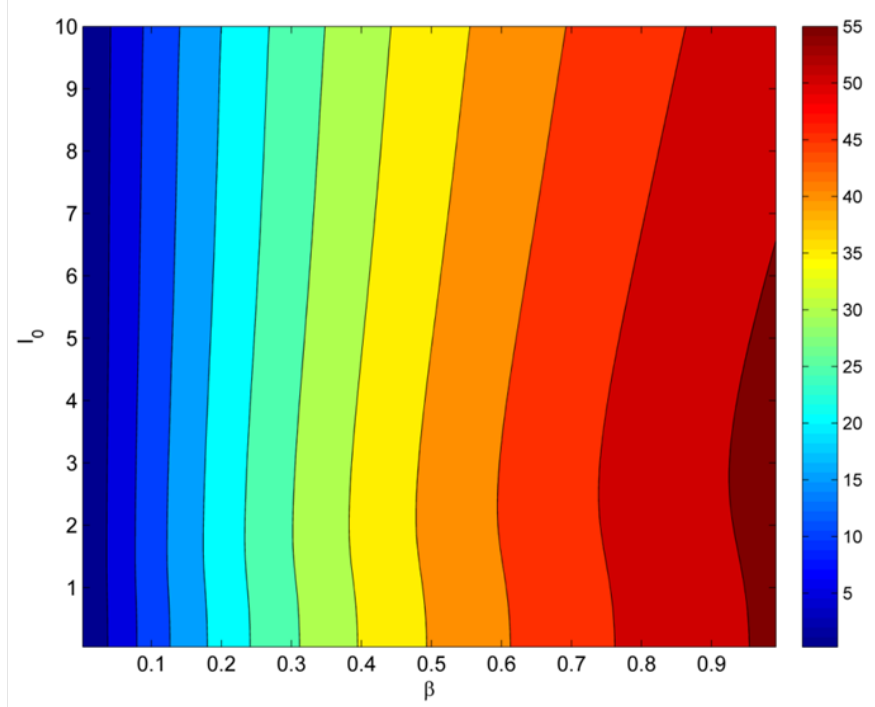

(b)

Figure 3: (a) Total nondimensional energy harvested and (b) energy harvesting efficiency for a simulation time of $\tau_{f}=110$ as a function of $\tilde{I}_{0}$ and $\beta$

which is expected for strongly nonlinear systems. Recall from Fig. 2 and the discussion in Section 3 that the initial energy state of the primary system dictates which orbits are "experienced" by the system. The work in $[26,27]$ indicated that high initial energy states under the initial conditions (19) correspond to high-frequency dynamic instabilities in the neighborhood of the upper IOM. These high-frequency transitions provide for good energy harvesting performance, and are thus predicted in this case to be the driving mechanism behind the response. The contour plot in Fig. 3a also indicates that total energy harvested depends moderately upon the electromechanical coupling parameter. The contour plot in Fig. 3b denotes the importance of the electromechanical coupling parameter, which strongly influences the energy harvesting efficiency. Plateaus of increasing efficiency exist for discrete ranges of electromechanical coupling and for the entire range of impulse magnitudes studied. It should be noted that these findings depend on the nondimensional time parameter $\tau$, or the time interval of computation for harvesting measures (21) and (22). It is intuitive that energy can continue to be harvested until the system comes to rest. Studying individual time histories at discrete points in these contours can provide more insight into 
the dependence on time.

The dynamics governing various regimes of the plots presented in Fig. 3 can be studied in detail by using wavelet analysis to analyze the nonlinear transient response of the system. High-frequency transient resonance captures are predicted for large impulse magnitudes and for any electromechanical coupling. The response of system (17) for a nondimensional impulse magnitude of $\tilde{I}_{0}=9$ is examined for system parameter values listed in Table 1 and electromechanical coupling $\beta=[0.1,0.9]$. As deduced from Fig. 3, these parameter combinations will provide insight into low and high harvesting efficiency regimes and for large total energy harvested. These responses are depicted in Fig. 4 and Fig. 5.

The response of the system for $\beta=0.1$ and $\tilde{I}_{0}=9$ is shown in Fig. 4. As seen in Fig. 3b and Fig. 4e, this system response corresponds to an overall energy harvesting efficiency of approximately $13 \%$, in which $7 \%$ of the harvesting occurs within the first $\sim 5$ simulation time units. Examination of the multi-frequency time history in Fig. 4c and relative displacement wavelet transform in Fig. 4d reveals the dynamics during the first $\sim 5$ simulation time units. As seen clearly in Fig. 4d, the transient response of the NES initially occurs in the neighborhood of high-frequency superharmonic tongues in the vicinity of the upper branch of the IOM. This is indicated by the dominant high-frequency harmonics in the initial, highly energetic phase of the relative response. As mentioned earlier, the primary system was scaled so that it inherently has a fundamental frequency of unity, as seen in Fig. 4a and Fig. 4b. Frequency transitions above unity correspond to motions in which the NES oscillates faster than the primary system, which is beneficial to energy harvesting. Following the high-frequency TRCs, the dynamics of the system transition to the lower-frequency $S 11+$ backbone branch, where energy is further harvested at a slower rate or dissipated via the viscous damping in the oscillators. To reiterate, these high-frequency TRCs are a consequence of the intentional strong cubic nonlinearity imposed in the coupling of the NES.

The response of the system for $\beta=0.9$ and $\tilde{I}_{0}=9$ is shown in Fig. 5, which exhibits high-frequency TRCs similar to those presented in Fig. 4. As seen in Fig. 5e, approximately $15 \%$ of the total $47 \%$ energy harvested occurs within the first $\sim 5$ simulation time units. Examination of the relative displacement wavelet in Fig. 5d clearly indicates that the dynamics tracks high-frequency TRCs in the neighborhood of the upper branch of the IOM within the first $\sim 5$ time units. Similar to the response depicted in Fig. 4, the 
(a)

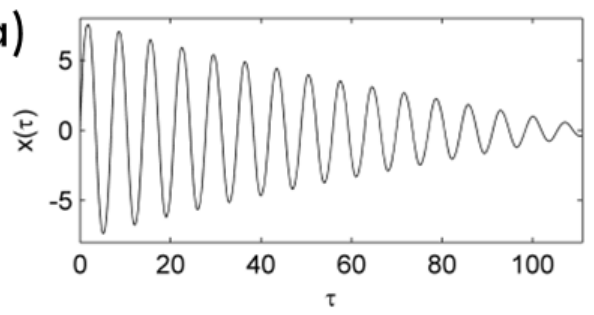

(c)

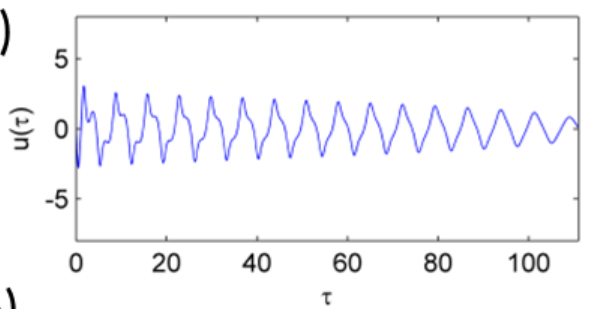

(e)

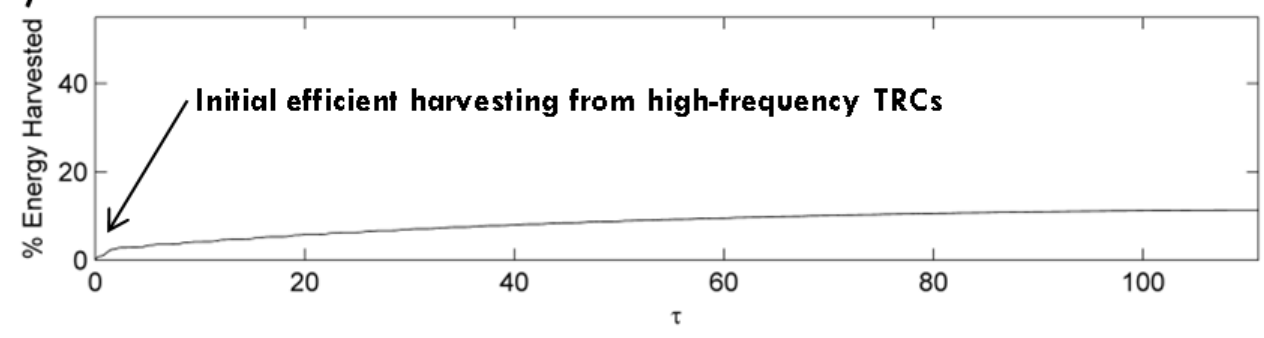

(b)

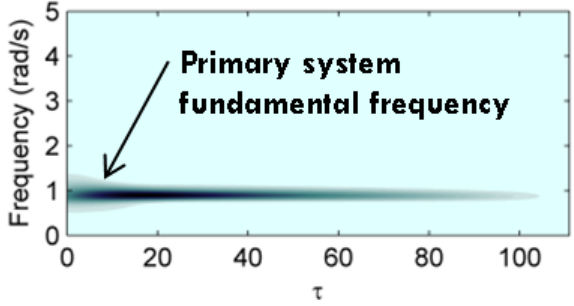

(d)

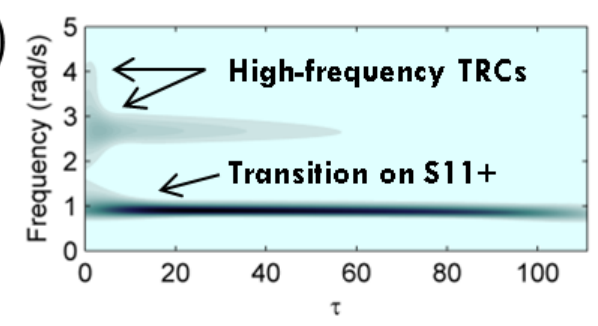

Figure 4: (a) Primary system displacement time history, (b) primary system displacement wavelet transform amplitude, (c) relative displacement time history, (d) relative displacement wavelet transform amplitude, and (e) energy harvesting efficiency measure (22) for system (17) with $\tilde{I}_{0}=9$ and $\beta=0.1$. 
(a)

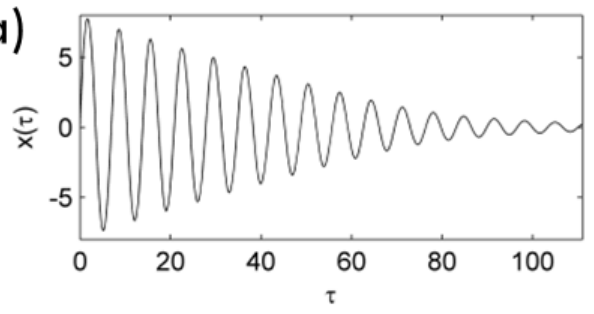

(c)

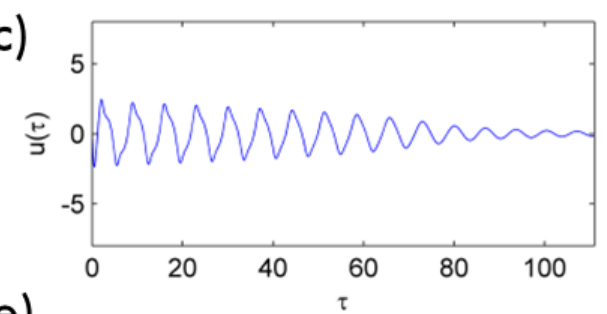

(e)

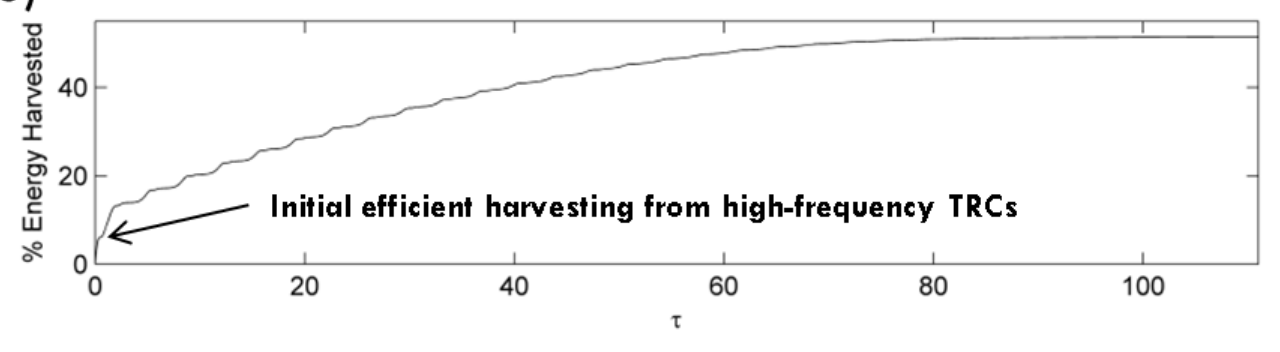

(b)

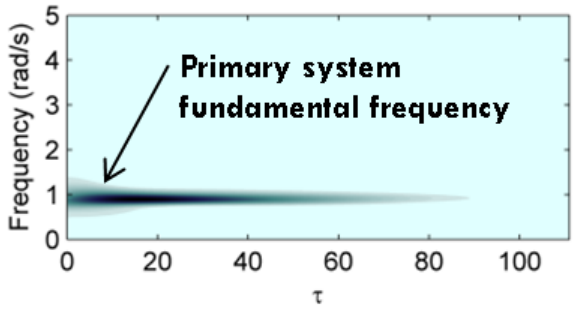

(d)

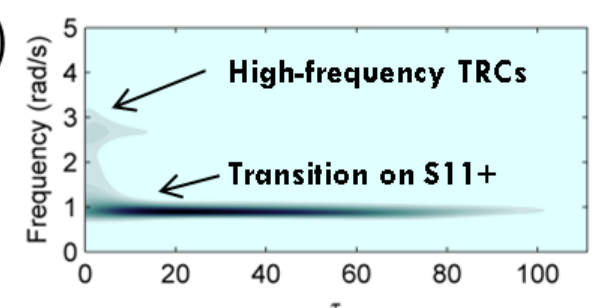


dynamics of the system transitions to the lower-frequency $S 11+$ backbone branch after the high-frequency TRCs die out. Energy is again passively dissipated and harvested at a slower rate until the system comes to rest. The effect of the increased electromechanical coupling in Fig. 5e is evident when that figure is compared to Fig. 4e. The total energy harvesting efficiency increases from $13 \%$ to $47 \%$ when the electromechanical coupling increases from 0.1 to 0.9 . In addition, the time to maximum obtained efficiency or steady system conditions decreases from 110 time units to 80 time units with increased electromechanical coupling. However, the high-frequency dynamic instability depends on the initial energy state of the system rather than the electromechanical coupling.

A similar analysis was carried out for lower impulse magnitude regions of the contour plots presented in Fig. 3. For impulse magnitudes $\tilde{I}_{0}<\sim 4$ and any electromechanical coupling $\beta$, the dynamics of the system initiates on and tracks the $S 11+$ backbone branch for the duration of the simulation. While the system remains in motion during this tracking, energy is harvested at a rate similar to that presented in Fig. 4e and Fig. 5e during $S 11+$ tracking. This defines an important general magnitude threshold for exciting high-frequency dynamic instability in the system, which is similar to results presented in $[26,27]$. This magnitude threshold can be rescaled to the physical primary system initial velocity value as $\dot{y}_{1}(0)=\tilde{I}_{0} \cdot c_{x} / c_{t}=I_{0}$, which indicates the initial energy level required to excite the experimental apparatus into high-frequency instability.

This previous analysis is important for the design and fabrication of the induction coil and selection of the permanent magnet, which determines the transduction factor $k_{e}$ and the electromagnetic damping $b_{e}$, and thus the nondimensional electromechanical coupling coefficient $\beta$. Physical limits are imposed on the transduction factor value via the size of the inductance coil and the magnetic field flux density inherent to the permanent magnet, as indicated in (8). As described earlier, the primary goal of this study is to experimentally demonstrate the effect of high-frequency dynamic instability on energy harvesting rather than strictly optimizing the system for harvesting energy, which provides some freedom in the design and fabrication of the induction coil. Detailed information regarding the inductance coil construction and permanent magnet selection will be presented in Section 6, however the analysis behind the construction and parameter identification will be discussed here.

The inductance coil used in the experimental apparatus (cf. Fig. 12) 

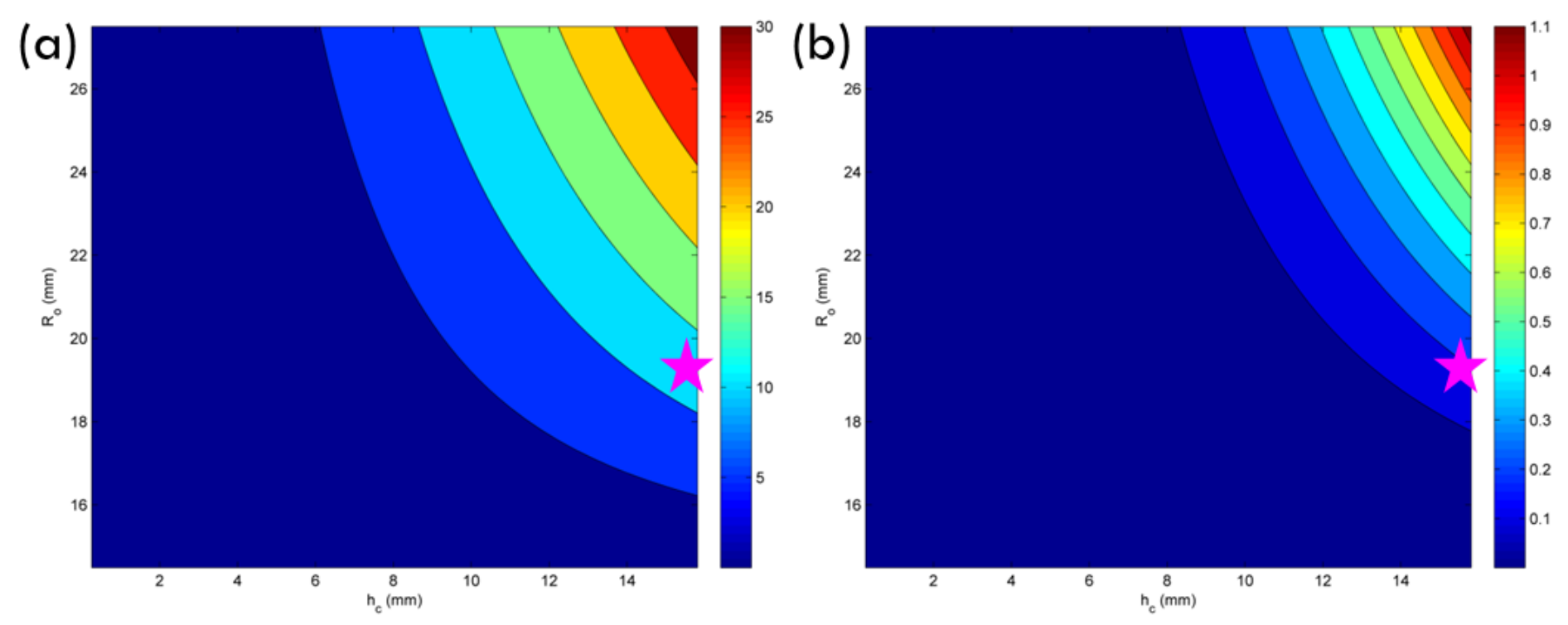

Figure 6: (a) Transduction factor (in Tesla-meters) and (b) nondimensional electromechanical coupling for an orthogonal fill factor inductance coil of thickness $h_{c}$ and outer radius $r_{o}$. The pink star denotes the targeted parameter values for the coil in the experimental apparatus.

Table 1: Nondimensional parameters for the system (17)

\begin{tabular}{ll}
\hline \hline Parameter & Value \\
\hline$\mu$ & 0.1850 \\
$\lambda$ & 0.0059 \\
$\zeta$ & 0.6653 \\
$\beta$ & 0.1018 \\
$\gamma$ & 0.0000 \\
\hline \hline
\end{tabular}


was wound by the authors assuming an orthogonal fill factor, as described in [28]. The fill factor encompasses tightness of winding, insulation thickness, and winding shape, and essentially determines the efficiency of the coil. This orthogonal fill factor corresponds to a coil efficiency of $\sim 80 \%$, and is obtainable practically with careful coil wrapping. Physically, the orthogonal fill factor describes coil winding in which each new turn of wire lies directly on top of the wire turn below it and perfectly in line with the wire turn next to it. Using this assumption, contour plots can be constructed to estimate the transduction factor (cf. Fig. 6a) and nondimensional electromechanical coupling parameter (cf. Fig. 6b) for specific coil dimensions. As seen in Fig. 6 , the outer radius and coil thickness of the wire wrapping can be varied to determine the parameter values, which are determined from (8) and (18). The minimum and maximum coil dimensions are constrained by the spool geometry used in the experimental apparatus.

As deduced from Fig. 6a, the transduction factor is strongly dependent upon the coil thickness relative to the coil radius. This indicates that the length (surface area) of the magnetic field induced by the coil is more important in the electromechanical coupling than the radial thickness of the magnetic field. Therefore the full thickness of the spool $(16 \mathrm{~mm})$ was wrapped when constructing the coil to maximize the transduction factor for a given length of wire, which leads to a given coil resistance and coil inductance. As seen in Fig. 6b, this also serves to maximize the electromechanical coupling parameter, which was investigated in Fig. 3. Recall from the derivation in Section 2 that the coil inductance $L_{c}(11)$ and resulting impedance $Z_{L}$ (12) can be estimated from the coil dimensions. In addition the coil inductance was assumed to be negligible in the analysis outlined following (13). Therefore the coil dimensions have self-imposed physical limitations to maintain the assumptions from Section 2.

From the analysis deduced from Fig. 3 and Fig. 4, a coupling parameter of $\beta=0.1$ was deemed acceptable to show the effects of high-frequency dynamic instability on energy harvesting capability while keeping the coil construction simple and maintaining the assumptions from Section 2. Therefore, as indicated by Fig. 6b, the coil must have a thickness of $16 \mathrm{~mm}$, inner radius of $14 \mathrm{~mm}$, and outer radius $\sim 20 \mathrm{~mm}$. This design is marked by a pink star in Fig. 6. The coil was wound with $N=819$ turns to achieve these dimensions. The coil resistance was measured with an ohmmeter, which can be seen in Table 2 . The coil inductance and impedance were determined from (11) and (12), respectively, assuming a maximum system frequency of $25 \mathrm{~Hz}$. 
The resulting reactance was indeed negligible relative to the coil resistance at $Z_{L}=0.1 \mathrm{~m} \Omega$.

The coil parameters were identified and confirmed experimentally by comparing a simple constrained experimental system to the corresponding numerical system. The permanent magnets were attached to an APS Dynamics ELECTRO-SEIS@Model 400 long-stroke shaker via a rigid rod, and the fully constructed induction coil was rigidly fixed to an optical table separate from the shaker. The magnets were aligned within the coil as they would be during the harvesting experiment, such that there would be a constant magnetic field for each half oscillation. Displacement data was collected for this constrained system with the shaker operating at $10 \mathrm{~Hz}$ and $1.5 \mathrm{~mm}$ displacement. Voltage data was collected across a load resistance of $R_{L}=47 \Omega$. The corresponding numerical system was constrained similarly for the same input conditions and load resistance. The transduction factor was varied until the numerical harvested energy was the same as the experimental harvested energy. The resulting transduction factor and electromagnetic damping parameters are given in Table 2. The final nondimensional electromechanical coupling parameter $\beta$ can thus be computed, and is shown in Table 1 . It should be noted that the electromechanical parameter values presented in Table 1 and below in Table 2 have strong correspondence to the targeted design parameter values presented in Fig. 6, validating the assumptions, design, and construction of the coil.

\section{Analytical Study}

To study the dynamics of this system analytically, a formal method of averaging is applied to the nondimensional equations of motion, with the transformations

$$
\begin{array}{rlrl}
x(\tau) & =r(\tau) \cos \phi(\tau), & u(\tau) & =\rho(\tau) \cos \theta(\tau), \\
x^{\prime}(\tau) & =-r(\tau) \sin \phi(\tau), \quad u^{\prime}(\tau)=-\rho(\tau) \Omega(\rho(\tau)) \sin \theta(\tau) .
\end{array}
$$

Note that the frequency-like quantity $\Omega$ depends explicitly only on $\rho$, the amplitude of the nonlinear attachment. Thus, $\Omega(\rho)$ describes the periodamplitude relation of the essentially nonlinear attachment, and will be determined as part of the solution procedure. With these transformations, the equations of motion in terms of the amplitude and phase variables can be 
written as

$$
\begin{aligned}
& r^{\prime}=x^{\prime} \cos \phi-x^{\prime \prime} \sin \phi \\
& \left(\Omega+\rho \Omega_{\rho} \sin ^{2} \theta\right) \rho^{\prime}=\Omega u^{\prime} \cos \theta-u^{\prime \prime} \sin \theta \\
& -r \phi^{\prime}=x^{\prime} \sin \phi+x^{\prime \prime} \cos \phi \\
& -\rho\left(\Omega+\rho \Omega_{\rho} \sin ^{2} \theta\right) \theta^{\prime}=\left(\Omega+\rho \Omega_{\rho}\right) u^{\prime} \sin \theta+u^{\prime \prime} \cos \theta
\end{aligned}
$$

where $\Omega_{\rho} \equiv \frac{d \Omega}{d \rho}$. From the equations of motion written in terms of these coordinates, the phase variable $\psi \equiv \theta-\phi$ can be identified with the $1: 1$ resonance between the linear primary system and the essentially nonlinear attachment. When the system vibrates in this resonant state the phase variable $\psi$ is, on average, stationary (i.e., non time-like varying), while outside of this condition $\psi$ is a time-like quantity. It is emphasized here that the existence of the TRCs considered in this work are directly related to the existence of the resonant phase difference $\psi$ in the neighborhood of a resonance manifold of the dynamics [30]. Application of the method of averaging to these equations of motion can uncover the coupling between the amplitudes $r$ and $\rho$, and the resonant phase $\psi$, and yields to following slow flow equations,

$$
\begin{aligned}
r^{\prime} & =-\frac{\lambda r}{2}-\frac{\mu}{2}\left\{(\beta+\xi) \Omega \rho \cos \psi+\frac{3}{4} \rho^{3} \sin \psi\right\} \\
r \phi^{\prime} & =r+\frac{\mu}{2}\left\{\frac{3}{4} \rho^{3} \cos \psi-(\beta+\xi) \Omega \rho \sin \psi\right\} \\
\left(\Omega+\frac{\Omega_{\rho} \rho}{2}\right) \rho^{\prime} & =-\frac{(1+\mu)}{2}(\beta+\xi) \Omega \rho+\frac{r}{2} \sin \psi-\frac{\lambda r}{2} \cos \psi \\
\left(\Omega+\frac{\Omega_{\rho} \rho}{2}\right) \rho \theta^{\prime} & =\frac{\Omega}{2}\left(\Omega+\Omega_{\rho} \rho\right) \rho+\frac{(1+\mu)}{2} \frac{3}{4} \rho^{3}+\frac{r}{2} \cos \psi+\frac{\lambda r}{2} \sin \psi
\end{aligned}
$$

with $\psi^{\prime} \equiv \theta^{\prime}-\phi^{\prime}$. Note that the equations have been written with $\theta^{\prime}$ and $\phi^{\prime}$ distinct to facilitate later comparison with numerical simulations of the original equations of motion. The period-amplitude dependence is incorporated by requiring that

$$
\Omega\left(\Omega+\Omega_{\rho} \rho\right)=(1+\mu) \frac{3}{4} \rho^{2} \quad \Longrightarrow \quad \Omega^{2}=(1+\mu) \frac{3}{8} \rho^{2} .
$$


Finally, the average power harvested becomes

$$
P_{\mathrm{h}}=\frac{\mu \beta}{2}(\Omega \rho)^{2}=\frac{\mu \beta(1+\mu)}{2} \frac{3}{8} \rho^{4} .
$$

For small primary damping $\lambda$ and mass ratio $\mu$, the evolution of $r$ described in Eq. (25a) is slow, while the time scale of the dynamics in the $\rho-\psi$ plane is $\mathcal{O}(1)$. Thus, we expect the general response of these averaged equations to be characterized by fast oscillations in $\rho$ and $\psi$, accompanied by slow drift in $r$. Moreover, a normally hyperbolic motion (NHM) can be identified in this general three-dimensional phase space that is, to lowest order, approximated by the equilibrium points in the $\rho-\psi$ equations for fixed $r$. When stable, the response of the system is attracted to this normally hyperbolic motion, so that the dynamics of the averaged system follows these slowly varying equilibria in the $\rho-\psi$ plane. In Fig. 7a,b the response of the averaged system to impulsive initial conditions is shown as a function of time. These are then combined in Fig. 7c to show the response in phase space. In addition to the trajectory in Fig. 7c shown in red, the slowly varying equilibria are shown, and both are projected on the sides of the figure box. We note that the phase variable $\psi$ is $2 \pi$-periodic, so that two copies of the slowly varying equibria are shown in black, differing only by a rotation. For these system parameters and initial conditions, the trajectory is initially attracted to the NHM, approximated by the slowly varying equilibria. As the system evolves, the amplitude of the primary system, given by $r$, decreases and the system continues to track the NHM until the branch of the slowly varying equilibria undergoes a saddle-node bifurcation in $r$. This saddle-node bifurcation is clearly seen in the projection onto the $r-\psi$ plane. The trajectory subsequently jumps in $\rho$ and $\psi$ to the continuation of the NHM, which occurs near $t=175$ in this simulation. This is accompanied by a complete rotation in $\psi$ before once again approaching the NHM.

For comparison of the above response to the averaged equations of motion, in Fig. 8 the response of the original equations of motion is shown with identical parameters. In this, the time series of $\left(x, x^{\prime}, u, u^{\prime}\right)$ have been transformed according to Eqs. (23) to obtain equivalent values of $\left(r_{\text {org }}, \rho_{\text {org }}, \psi_{\text {org }}\right)$, which correspond to the previously identified averaged variables. Note, however, that these transformations do not introduce any kind of averaging into the resulting time series. The response of the averaged equations shown in Fig. 9 is similar to that of the original equations of motion, illustrated in Fig. 8. In particular, we note the response of the original equations of 
(a)
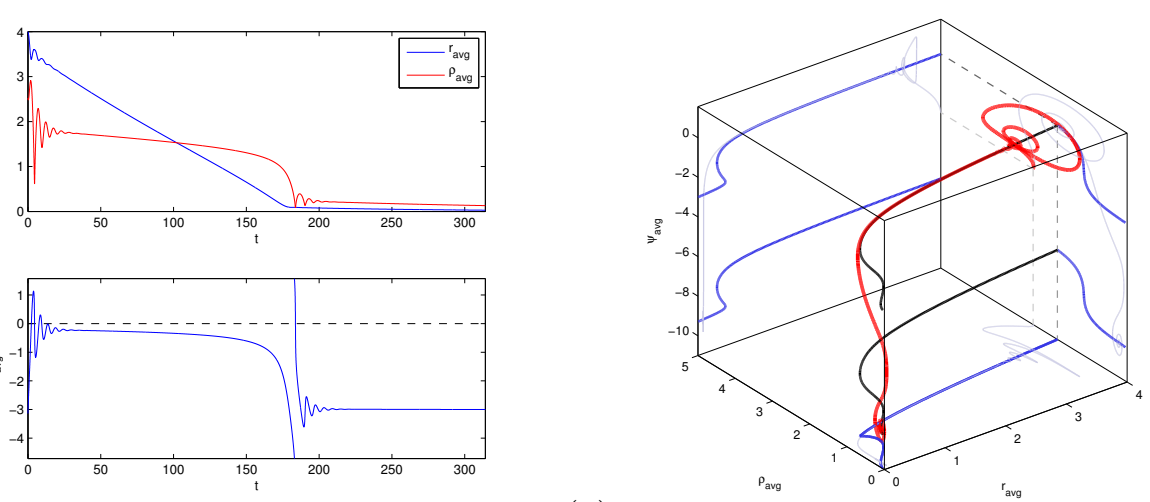

(b)

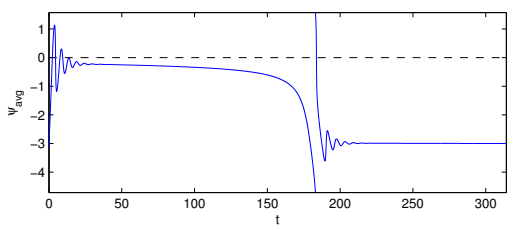

(c)

Figure 7: Response of the averaged equations of motion $\left(I_{0}=4.00, \mu=0.05, \lambda=0.01\right.$, $\xi=0.10, \beta=0.25$ ). In panel (c) the projections of the response and slowly varying equilibria are shown.

motion oscillates on a fast time scale about the slowly-varying equilibria identified in the averaged equations of motion, so that the equivalent coordinates $\left(r_{\text {org }}, \rho_{\text {org }}, \psi_{\text {org }}\right)$ are useful for describing the response of the original equations of motion.

Similar results, corresponding to the experimentally identified parameter values, are shown in Fig. 9 and 10. In particular, the numerical simulations of the original equations of motion correspond to the time series shown in Fig. 4. Despite the much larger mass ratio identified in the experimental system $(\mu=0.185)$ there is qualitative agreement between the response from the original and averaged equations of motion. In particular, as the trajectories evolve they follow the slowly varying equilibria identified from the averaged equations of motion. We note, however, that the time evolution of the two responses differs primarily in the evolution of $r$, the amplitude of the primary system. The original equations of motion show increased dissipation during the initial interval of motion. This enhanced energy transfer from the primary system can be attributed to the high-frequency transient resonance capture identified from the frequency-energy plots shown in Fig. 4.

Finally, in Fig. 11 the slowly varying equilibria are shown for fixed $\beta$ as the primary amplitude $r$ varies. In Fig. 11a, b this curve is shown for a single value of $\beta$, while the resulting surface, formed by a continuation of $\beta$ is illustrated in Fig. 11c. For sufficiently small $\beta$, this curve undergoes a pair of saddle-node bifurcations as $r$ varies, as shown in Fig. 11a. In particular, 
(a)

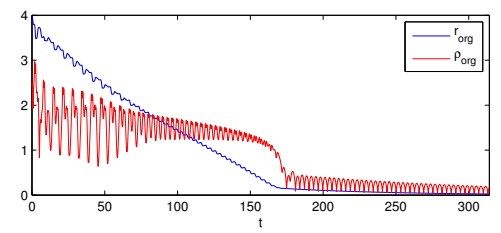

(b)

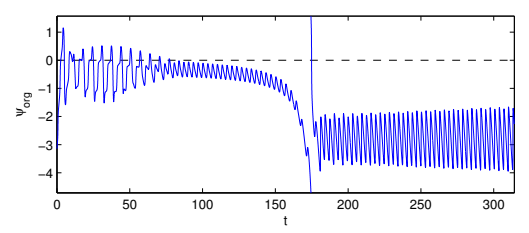

(c)

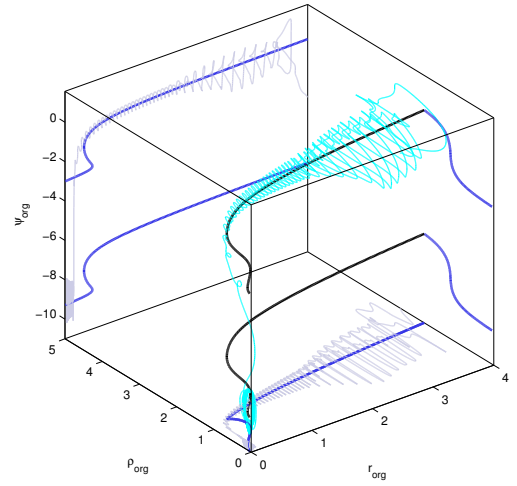

Figure 8: Response of the original equations of motion, Eqs. (17) $\left(I_{0}=4.00, \mu=0.05\right.$, $\lambda=0.01, \xi=0.10, \beta=0.25$ ). In panel (c) the projections of the response and slowly varying equilibria are shown.

(a)

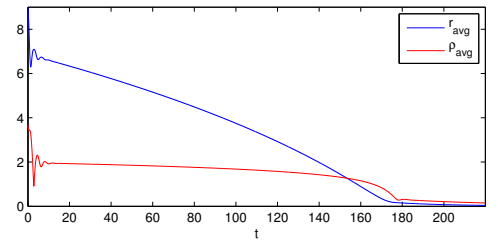

(b)

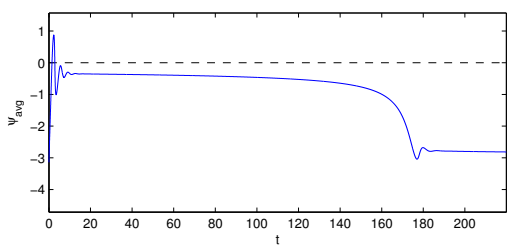

(c)

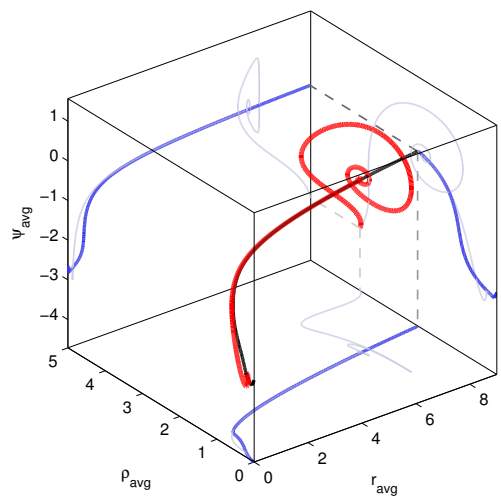

Figure 9: Response of the averaged equations of motion $\left(I_{0}=9.00, \mu=0.185, \lambda=0.0059\right.$, $\xi=0.6653, \beta=0.1018)$. In panel (c) the projections of the response and slowly varying equilibria are shown. 
(a)

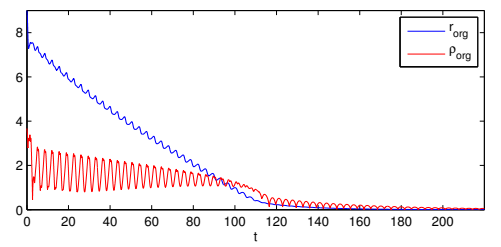

(b)

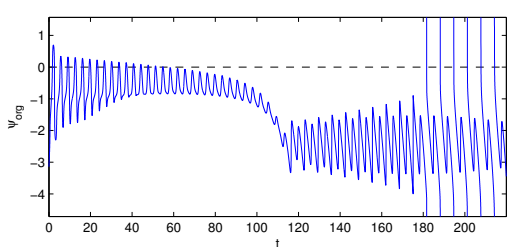

(c)

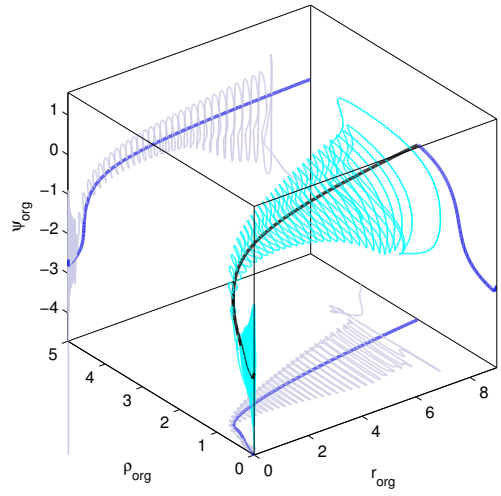

Figure 10: Response of the original equations of motion, Eqs. (17) $\left(I_{0}=9.00, \mu=0.185\right.$, $\lambda=0.0059, \xi=0.6653, \beta=0.1018)$. In panel (c) the projections of the response and slowly varying equilibria are shown.

there exists a branch of slowly varying equilibria that is sustained with large $\rho$. Trajectories along this large amplitude branch correspond to efficient energy harvesting (see Eq. (27)). These saddle-node bifurcations disappear for values of $\beta$ above a critical value, $\beta_{\text {cr }}=0.45$ shown in Fig. 11b. Above this value $\beta=\beta_{\text {cr }}$ no saddle-node bifurcations exist and the amplitude $\rho$ decreases continuously as $r$ decreases, as illustrated in the projection shown in Fig. 11d.

\section{Experimental Study}

The experimental apparatus is presented in Fig. 12. The primary system is denoted in the figure as the linear oscillator (HDPE mounting mass), piano wire (aluminum cross-bar beam and steel wire), and inductance coil (copper coil and aluminum mount). The primary system is grounded to an optical table via two thin steel rectangles, which provide for the linear grounding stiffness and light viscous damping. The lightweight nonlinear attachment (NES) is composed of the permanent magnets, collars, and a steel rod, which is supported by two linear roller bearings, one in each aluminum upright at the ends of the HDPE mounting mass. Additional linear viscous damping in the coupling arises from the interaction of the rod with the bearings. The NES is coupled to the primary system via a piano wire of diameter $0.5 \mathrm{~mm}$, which is physically attached to the NES via the collars. These 
(a)

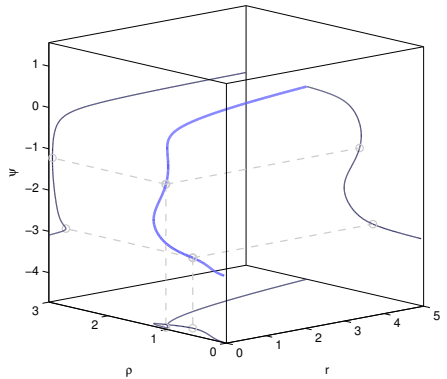

(c)

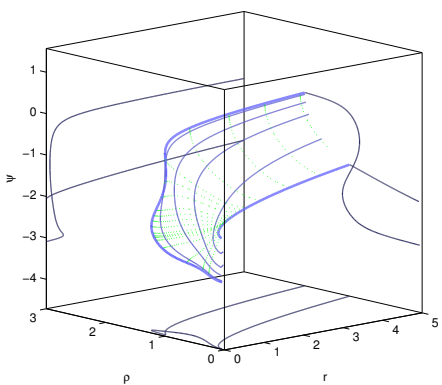

(b)

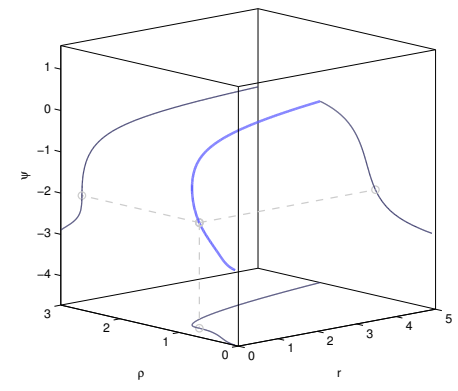

(d)

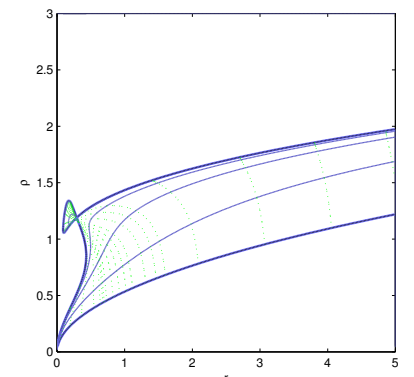

Figure 11: Slowly varying equilibra $(\mu=0.05, \lambda=0.01, \xi=0.10)$, each solid curve corresponds to a curve for fixed $\beta$; (a) $\beta=0.01$, (b) $\beta=0.45$, (c, d) $\beta=$ $[0.01,0.09,0.45,1.01,2.24,5.00]$. In panels $(\mathrm{a}, \mathrm{b})$ the projections of the curve are shown, while for reference in (c) only the projections corresponding to $\beta=0.01$ and $\beta=5.00$ are shown. The saddle-node bifurcations that occur as $r$ varies are marked in panels (a) and (b). 


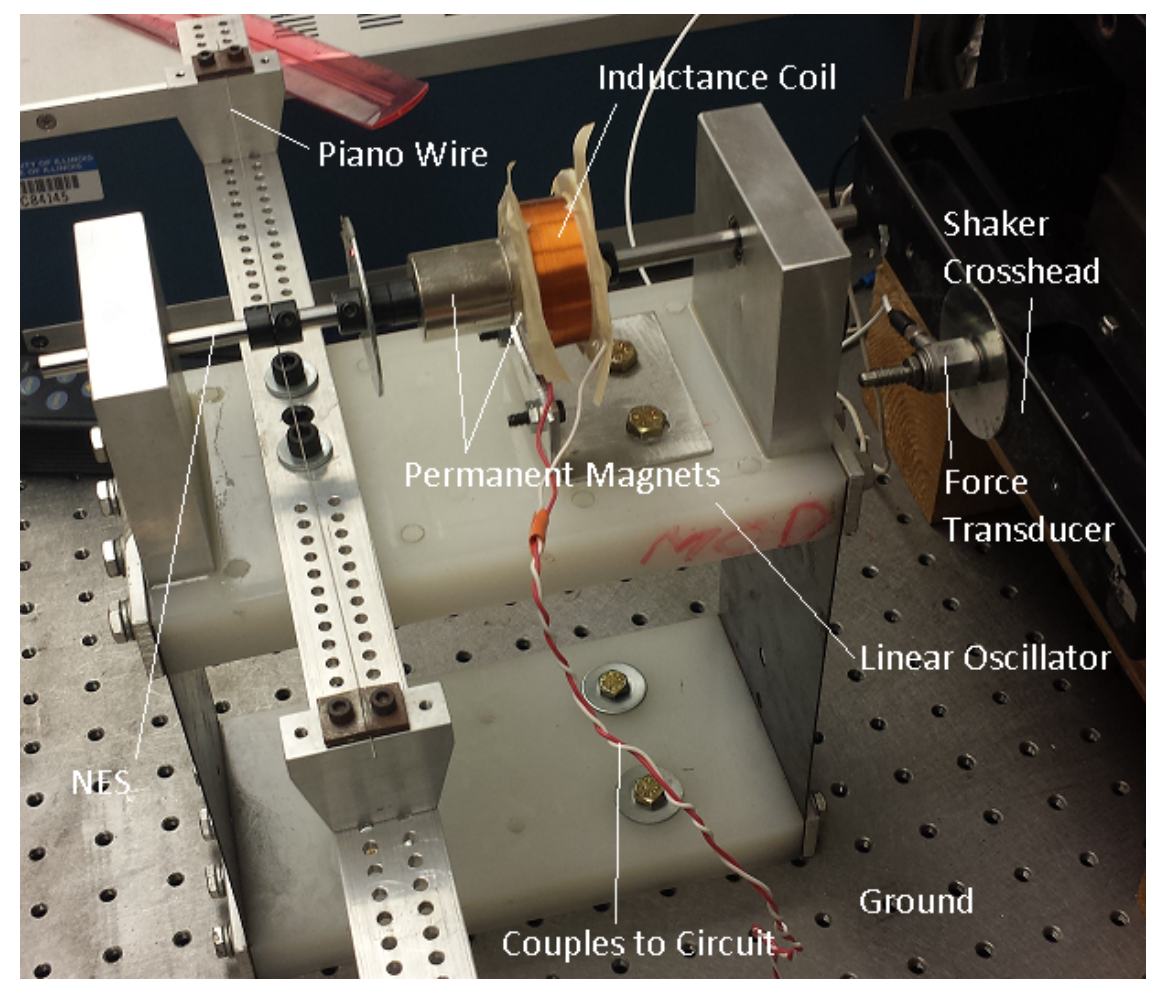

Figure 12: Experimental realization of the system depicted in Fig. 1. 
collars also serve to precisely hold the permanent magnets in place. As seen in Fig. 12, the piano wire is oriented perpendicular to the direction of motion of the attachment and the resulting transverse deflection gives rise to a dominant third-order, essential stiffness nonlinearity (a purely cubic nonlinearity) [30]. The piano wire is mounted in the cross-bar beam such that there is no pretension in the wire, minimizing any linear components in the coupling stiffness.

The inductance coil is constructed within a HDPE spool of inner radius $r_{i}=14 \mathrm{~mm}$. As described in Section 4, the coil is wrapped with $N=$ 819 turns to achieve a thickness of $h_{c}=16 \mathrm{~mm}$ and an outer radius of $r_{o}=20 \mathrm{~mm}$, which provides the desired electromechanical coupling. The coil was wound by the authors while striving to maintain the orthogonal fill factor, as described in the last section. Enameled AWG 30 copper wire is used to maximize turns while minimizing coil resistance. Two cylindrical neodymium (NdFeB) permanent magnets of dimensions $25.4 \mathrm{~mm}$ outside diameter and $25.4 \mathrm{~mm}$ length are used to create a uniform magnetic field within the coil. Neodymium magnets provide a strong magnetic field flux density for their size; the selected magnets provided a flux density of $B=$ $1.32 \mathrm{~T}$. The coil leads are connected to a breadboard and placed in series with a simple resistor, which functions as the load resistance for computing energy harvesting capability. The experimentally identified parameters for the physical apparatus from Section 4 are summarized in Table 2. This system is modeled numerically as described by (15).

Impulsive forces are applied to the linear oscillator by use of an instrumented PCB 086D20 modal hammer with a hard plastic tip with the system initially at rest, striving to replicate the initial conditions imposed in (19). Absolute velocity time series measurements of the two oscillators are recorded using two Polytec PSV laser vibrometers with a sampling frequency of $S_{F}=8.192 \mathrm{kHz}$ and a time period of $t_{f}=2.0 \mathrm{~s}$. Voltage time series measurement is taken across the load resistance, which could later be used to compute output power from the harvesting elements. The data acquisition is synchronized using the modal hammer as the trigger to start acquisition, with a small pretrigger time of $196 \mathrm{~ms}$. This synchronized measurement of the response of the system is important for accurate computation of the relative displacement wavelet spectra by eliminating any phase lag in the measurements. The raw velocity time series data is numerically integrated during post-processing to obtain absolute displacement time series data for each of the oscillators. The wavelet spectra are computed during post-processing 
Table 2: Dimensional parameters for the experimental apparatus described by system (15)

\begin{tabular}{ll}
\hline \hline Parameter & Value \\
\hline$m_{1}$ & $1.535 \mathrm{~kg}$ \\
$b_{1}$ & $0.480 \mathrm{Ns} / \mathrm{m}$ \\
$k_{1}$ & $4300 \mathrm{~N} / \mathrm{m}$ \\
$m_{2}$ & $0.284 \mathrm{~kg}$ \\
$b_{2}$ & $10.0 \mathrm{Ns} / \mathrm{m}$ \\
$k_{2}$ & $322,647 \mathrm{~N} / \mathrm{m}$ \\
$h$ & $0.133 \mathrm{~m}$ \\
$b_{e}$ & $1.53 \mathrm{Ns} / \mathrm{m}$ \\
$k_{e}$ & $11 \mathrm{Tm}$ \\
$R_{c}$ & $32.1 \Omega$ \\
$R_{L}$ & $47 \Omega$ \\
\hline \hline
\end{tabular}

from the time series data similarly to what was done in the computation study.

A series of ten experimental trials were conducted using the apparatus and measurement scheme described above. The trials covered a wide range of excitation magnitudes corresponding to the magnitude range explored in the nondimensional system (17) from Section 4. This range spanned from $I_{0}=[0.5,1.2] \mathrm{m} / \mathrm{s}$, which was determined from the maximum velocity of the primary system directly following the impulse from the modal hammer. The average width of the half-sine pulse provided from the modal hammer was $\sim 1.8 \mathrm{~ms}$. Recall that the forcing in the nondimensional system (17) was assumed to be impulsive in nature, so a minimized pulse width from the hammer excitation was important in maintaining this assumption in the numerical system.

Recall from Section 1 that the work to follow in this series will expand the following results into a study of this system under repeated impulse excitation to maintain energy harvesting from high-frequency instability. To enhance this effort, the following experimental results are compared to the corresponding numerical system (15). The corresponding numerical system is simulated using the forcing data from the modal hammer in the experimental trials as a means to validate the parameter identification from Section 4. The numerical system is further explored using the impulsive forcing as- 

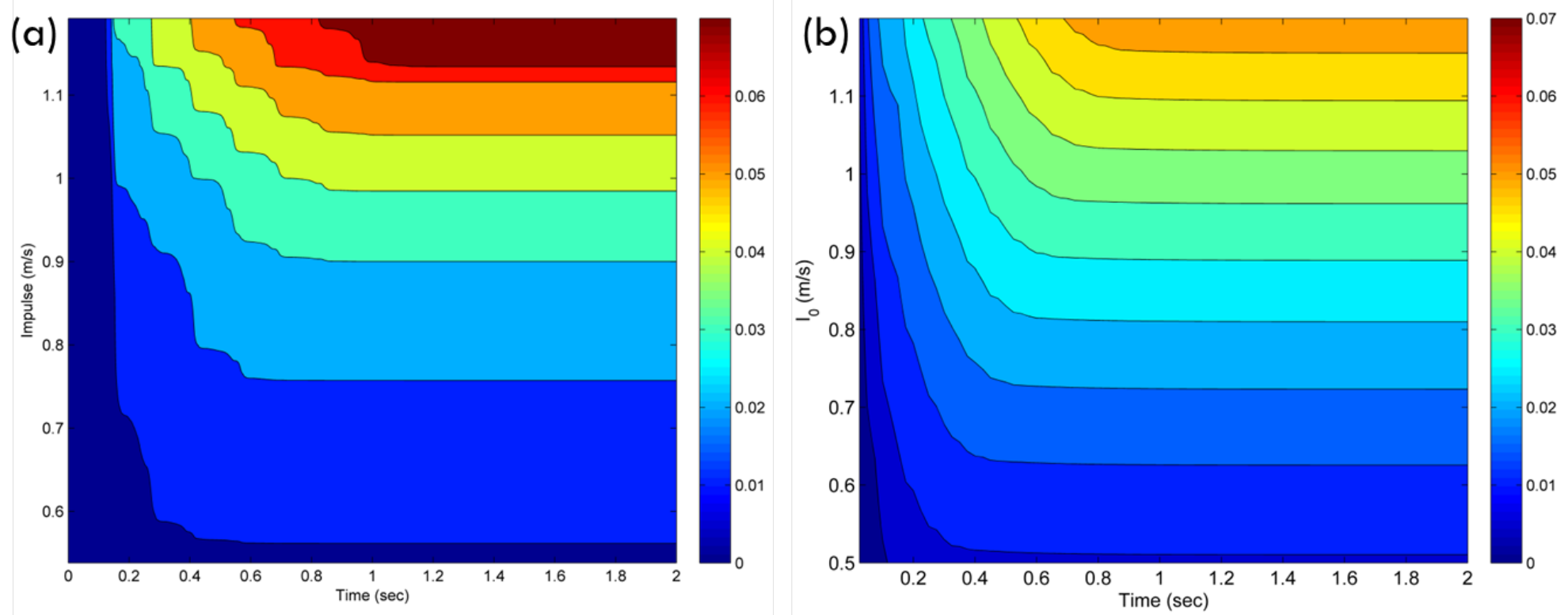

Figure 13: (a) Experimental and (b) numerical energy harvesting performance over time for various impulse magnitudes. Energy harvesting performance evaluated from (21) is presented in Joules.

sumption, as is done for the nondimensional system (17) in Section 4. This will allow for accurate exploration of the numerical system (15) forced by repetitive impulses in the next work if there is good correspondence between the experimental and numerical responses.

Performance contour plots for the system (15) are developed experimentally and numerically (impulsive forcing) using energy harvesting measure (21) reported in Joules. These results are presented in Fig. 13. While Fig. $13 \mathrm{~b}$ is constructed "smoothly" for impulse values discretized as $\Delta I_{0}=0.01$ $\mathrm{m} / \mathrm{s}$ for the whole range presented, there is some degree of interpolation in the data presented in Fig. 13a due to a larger discretization $\Delta I_{0}$ from performing only ten experimental trials. This figure is analyzed by focusing on horizontal "slices" in the contour and deducing the rate at which energy is being harvested. The pretrigger imposed in the data collection accounts for the first $\sim 0.2 \mathrm{~s}$ of no harvesting in Fig. 13a, whereas the impulsive forcing used in Fig. 13b initiates harvesting at the onset of the simulation. As deduced from Fig. 13, the harvesting performance (21) from the experi- 

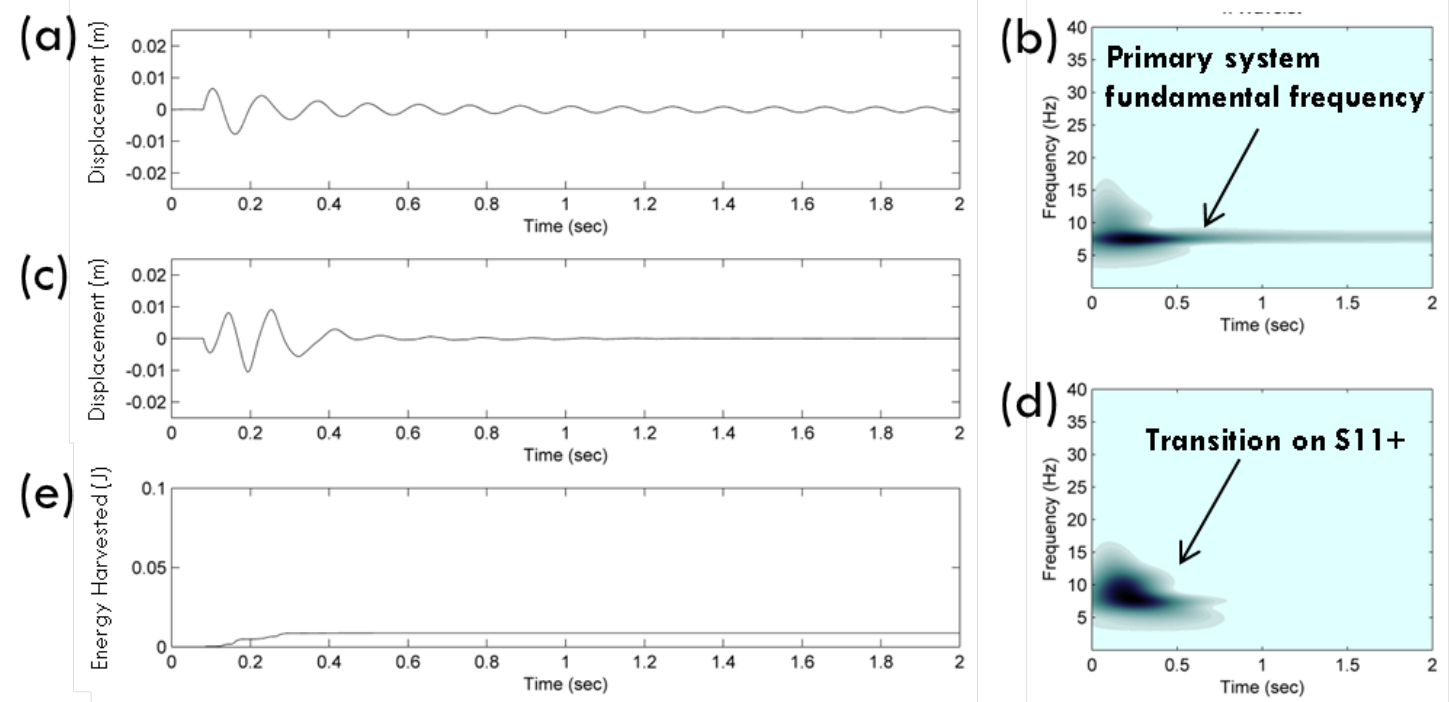

Figure 14: (a) Primary system displacement time history, (b) primary system displacement wavelet transform amplitude, (c) relative displacement time history, (d) relative displacement wavelet transform amplitude, and (e) energy harvesting performance measure (21) for the experimental apparatus presented in Fig. 12 subject to low intensity impulsive excitation of magnitude $I_{0}=0.54 \mathrm{~m} / \mathrm{s}$ from modal hammer.

mental apparatus has strong qualitative and quantitative correspondence to the harvesting performance from the numerical system (15). As seen in Fig. 13a, the apparatus harvests more energy at a faster rate for higher impulse magnitudes, which was predicted in Section 4 (cf. Fig. 3 and Fig. 5). The contour in Fig. 13a indicates a maximum experimentally harvested energy of $\sim 70 \mathrm{~mJ}$ over a duration of $\sim 1.0 \mathrm{~s}$, providing an average output power of $\sim 70 \mathrm{~mW}$. It is again predicted that this superior performance at higher energy levels is due to the presence of the high-frequency TRCs in the response of the system. For these large impulse excitations, the system harvests energy for $\sim 1.0 \mathrm{~s}$ before coming to rest, which is indicated experimentally and numerically by Fig. 13. The performance of the experimental apparatus can be analyzed further by investigating the time series and wavelet spectra for individual trials.

The response of the experimental apparatus shown in Fig. 12 for a low impulse intensity of $I_{0}=0.54 \mathrm{~m} / \mathrm{s}$ is presented in Fig. 14. As seen in Fig. $14 \mathrm{a}, \mathrm{b}$, the primary system oscillates at its fundamental frequency of $8.4 \mathrm{~Hz}$ 

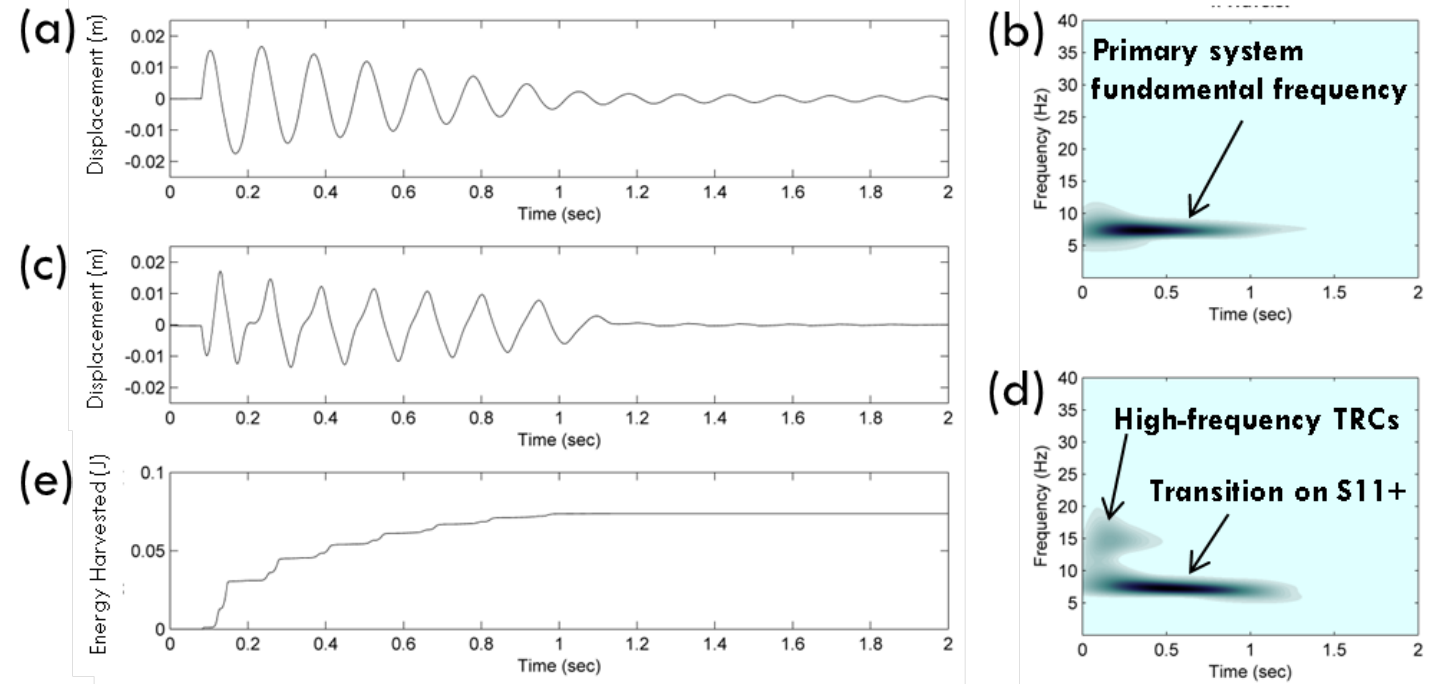

Figure 15: (a) Primary system displacement time history, (b) primary system displacement wavelet transform amplitude, (c) relative displacement time history, (d) relative displacement wavelet transform amplitude, and (e) energy harvesting performance measure (21) for the experimental apparatus presented in Fig. 12 subject to high intensity impulsive excitation of magnitude $I_{0}=1.19 \mathrm{~m} / \mathrm{s}$. from modal hammer

before eventually coming to rest. As seen in Fig. 14c,d, the NES simply engages in 1:1 internal resonance capture with the primary system before coming to rest after $\sim 0.4 \mathrm{~s}$. The energy input into the linear oscillator isn't large enough to excite the high-energy solutions presented in Fig. 2. The apparatus exhibits poor energy harvesting performance in this case, as seen in Fig. 14e. A maximum energy of $11 \mathrm{~mJ}$ is harvested slowly for a duration of $\sim 0.4 \mathrm{~s}$, resulting in an average power of $\sim 27.5 \mathrm{~mW}$. This performance is predicted in the nondimensional study presented in Section 4, which is confirmed here experimentally.

A much different response is obtained for stronger applied impulses. The response of the experimental apparatus for a high impulse intensity of $I_{0}=$ $1.19 \mathrm{~m} / \mathrm{s}$ is presented in Fig. 15. Similar to the low-intensity impulse shown in Fig. 14a, the primary system oscillates at its fundamental frequency before coming to rest, as indicated by Fig. 15a,b. This behavior is predicted by the nondimensional system (17) for any magnitude impulse excitation. This indicates that the attachment doesn't affect the underlying dynamics of the 
primary system, rather just the rate at which energy is removed from the primary system. As seen in the relative displacement time series in Fig. $15 \mathrm{c}$ and more clearly in the relative-displacement wavelet amplitude in Fig. $15 \mathrm{~d}$, the NES initially engages in high-frequency TRCs with the primary system in the neighborhood of the upper branch of the IOM within the first $\sim 0.4 \mathrm{~s}$. This high-frequency TRC occurs in the neighborhood of the 2:1 superharmonic internal resonance tongue, as indicated by the frequency content of $\sim 16 \mathrm{~Hz}$ in Fig. 15d. The corresponding energy harvested during the first $\sim 0.4 \mathrm{~s}$ can be deduced from Fig. 15e, which indicates $55 \mathrm{~mJ}$ of energy is harvested with a resulting average power of $\sim 137.5 \mathrm{~mW}$. The initial energy input into the primary system is now large enough to excite the high-energy solution presented in Fig. 2. As energy is removed via harvesting and passive dissipation, the system cannot continue operating in the high-frequency solution regime, which is indicated by Fig. 15d. As seen in the figure, the NES transitions to the $S 11+$ branch after $\sim 0.4 \mathrm{~s}$, engaging in 1:1 internal resonance capture with the primary system. Similar to the performance presented in Fig. 14d,e, energy is harvested at a slower rate for an additional $\sim 0.7 \mathrm{~s}$ during the 1:1 internal resonance capture, as deduced from Fig. 15d,e. A maximum energy of $72 \mathrm{~mJ}$ is harvested for a total duration of $1.1 \mathrm{~s}$, resulting in a total average power of $65.5 \mathrm{~mW}$. This performance is predicted in the nondimensional study presented in Section 4, which is confirmed here experimentally. This increased energy harvesting performance during high-frequency TRCs is the primary goal of this study and is now confirmed here for a novel experimental apparatus.

As discussed earlier, a secondary goal of this study is to validate the identified parameters from Table 2. This is accomplished in the analysis that follows by comparing the experimental system response presented in Fig. 15 with the corresponding numerical system (15) response utilizing the experimental forcing data. The system (15) is integrated numerically until $t_{f}=2.0 \mathrm{~s}$ using the large impulse intensity of $I_{0}=1.19 \mathrm{~m} / \mathrm{s}$, which is presented in Fig. 16. Similar to the experimental apparatus, Fig. 16a,b indicates that the primary system oscillates at its fundamental frequency before coming to rest. Again similar to the experimental apparatus, Fig. 16c,d indicates that the NES initially engages in high-frequency TRCs with the primary system in the neighborhood of the upper branch of the IOM within the first $\sim 0.3 \mathrm{~s}$. The transitions depicted in Fig. $15 \mathrm{~d}$ and Fig. $16 \mathrm{~d}$ vary slightly in that the experimental system shows dominant transition on the 2:1 superharmonic tongue while the numerical system shows transitions 
(a)

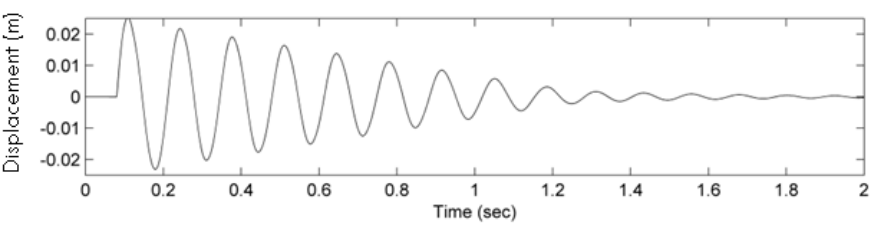

(c)

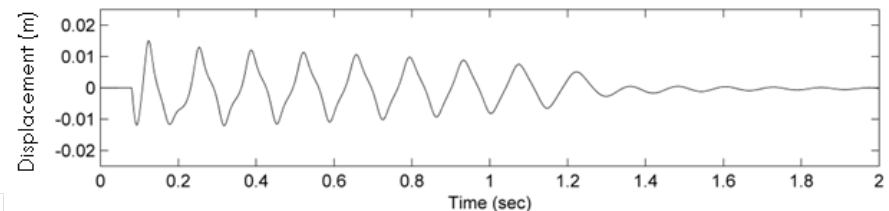

(e)

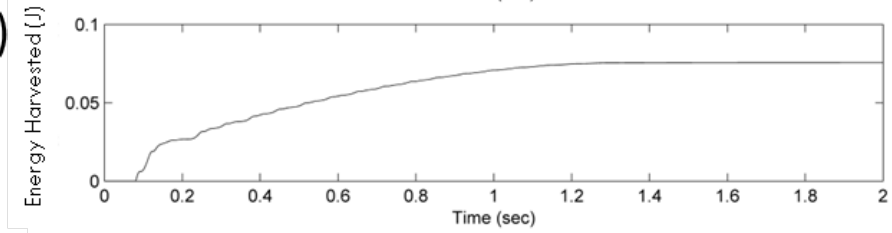

(b) ${ }_{35}^{40}$ Primary system

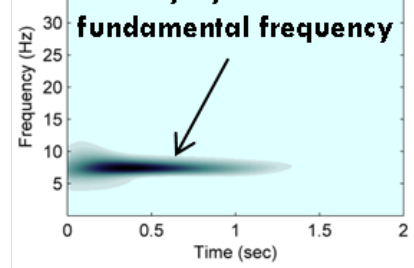

(d) ${ }_{35}^{40}$ High-frequency TRCs

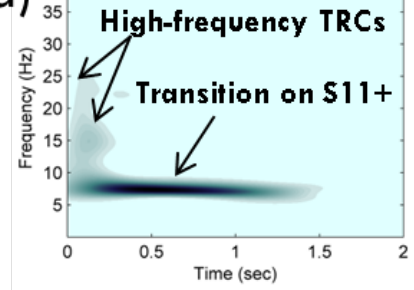

Figure 16: (a) Primary system displacement time history, (b) primary system displacement wavelet transform amplitude, (c) relative displacement time history, (d) relative displacement wavelet transform amplitude, and (e) energy harvesting performance measure (21) for the numerical system (15) subject to high intensity impulsive excitation of magnitude $I_{0}=1.19 \mathrm{~m} / \mathrm{s}$ provided from experimental data collected from the modal hammer. Figure generated using numerical simulation with experimental forcing data. 

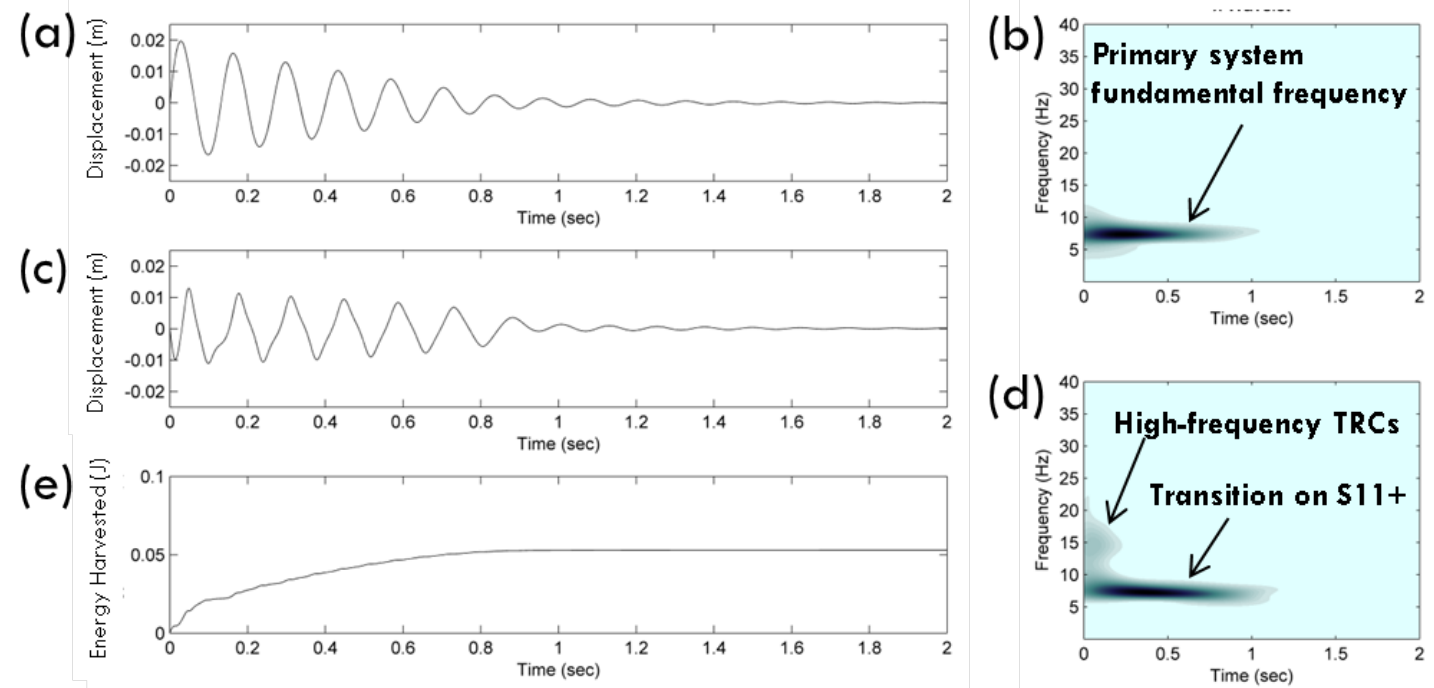

Figure 17: (a) Primary system displacement time history, (b) primary system displacement wavelet transform amplitude, (c) relative displacement time history, (d) relative displacement wavelet transform amplitude, and (e) energy harvesting performance measure (21) for the numerical system (15) subject to high intensity impulsive excitation of magnitude $I_{0}=1.19 \mathrm{~m} / \mathrm{s}$ provided from a pulse of Dirac nature. Figure generated using purely numerical simulation.

near the 3:1 and 2:1 superharmonic tongues within the first $\sim 0.3 \mathrm{~s}$. This slight variation in TRCs doesn't last long enough to have a profound effect on the energy harvested before the NES transitions to the $S 11+$ branch, as indicated by the similar energy harvesting capability in Fig. 15e and Fig. 16e. Comparing Fig. 15a and Fig. 16a, it can be seen that the numerical system experiences higher initial displacements than the experimental system for the first few cycles. The passive viscous damping in the experimental apparatus my be slightly greater than that identified and used in the numerical system, allowing for different frequency transitions. This could also be the effect of Coulomb friction present in the experimental system between the bearing and rod interface. Nevertheless, it is concluded here that the identified parameter set presented in Table 2 is validated. Numerical simulations of system (15) utilizing lower intensity impulse data from the experiments continues to show good quantitative and qualitative correlation with the results.

The final goal of this study is to extend these results, which entails sub- 
jecting this system to a series of repeated impulses. The authors seek to accurately explore system (15) numerically using a series of impulses and thus construct a baseline for future experiments. It must be shown that the impulse assumption is a good approximation of the experimental forcing. This is accomplished in the analysis that follows by comparing the experimental system response presented in Fig. 15 with the corresponding numerical system (15) response utilizing impulsive forcing. The system (15) is integrated numerically for $t_{f}=2.0 \mathrm{~s}$ using the large impulse intensity of $I_{0}=1.19 \mathrm{~m} / \mathrm{s}$, which is presented in Fig. 17. Similar to the experimental apparatus, Fig. 17a,b indicates that the primary system oscillates at its fundamental frequency before coming to rest. Again similar to the experimental apparatus, Fig. $17 \mathrm{c}, \mathrm{d}$ indicates that the NES initially engages in high-frequency TRCs with with the primary system in the neighborhood of the upper branch of the IOM within the first $\sim 0.2 \mathrm{~s}$. The TRCs depicted in Fig. 15d and Fig. 17d are similar in that both exhibit dominant transitions on the 2:1 superharmonic tongue, but the transitions vary in length of time captured. Fig. 17d depicts a faster transition to the 1:1 internal resonance branch than that presented in Fig. 15d. This is most likely due to the difference in actual energy input into the system between the two forcing schemes. The excitation magnitude is based on the maximum velocity of the linear oscillator directly following the impulse, rather than the energy in the actual transmitted pulse. Dirac forcing assumes an infinitesimally small pulse width, while the experimental forcing has an average pulse width of $\sim 1.8 \mathrm{~ms}$. Therefore the energy imparted into the system due to the impulsive forcing is conservative compared to the energy imparted into the system due to the experimental forcing from the modal hammer. This is evident in the overall lower energy harvested from the impulsive forcing (cf. Fig. 17e) when compared to the overall higher energy harvested from the experimental apparatus (cf. Fig. 15e). The characteristic increase in energy harvesting performance during high-frequency TRC is still present in Fig. 17e for the impulsive forcing, which is most important for these purposes. Nevertheless, it is concluded here that the impulsive forcing scheme is an acceptable approximation of the experimental forcing from the modal hammer, with the energy consideration taken into account. Numerical simulations of system (15) utilizing lower intensity impulses continues to show good quantitative and qualitative correlation with the experimental results. 


\section{Conclusions}

This investigation presented an analytical, computational, and experimental study of the single-impulsive dynamics of a strongly nonlinear electromechanical energy harvester. The system is composed of a directly excited linear primary oscillator coupled to a lightweight attachment (NES) through a strongly nonlinear cubic stiffness element and electromagnetic harvesting elements. This strong nonlinearity gives rise to high-frequency nonlinear transient resonance captures in the damped dynamics of the two oscillators. These high-frequency TRCs cause dynamic instabilities that lead to large-amplitude relative displacements between the oscillators. These largeamplitude solutions provide for increased energy harvesting capability.

The work showed numerically and experimentally that for an appropriate (although non-optimized) harvester design the high-frequency TRCs result in strong energy harvesting performance relative to that occurring at 1:1 resonance conditions. This was done by comparing the energy harvested and power output in a novel experimental apparatus. Wavelet spectral analysis was used to indicate the presence of these high-frequency TRCs. Furthermore, the analytical studies, developed by averaging the equations of motion, identify the underlying structure of the phase space that guides the damped dynamics.

The study compared numerical and experimental responses to validate the experimental parameter identification, with minimal error. Finally, these identified parameter values were used to show qualitative agreement between the dynamics of the averaged and original equations of motion. Comparison

of numerical and experimental responses also provided a baseline to extend these results to a companion work, which evaluates this system subject to repeated impulses.

\section{Acknowledgment}

This work was supported in part by National Science Foundation Grant No. CMMI-1100722.

\section{References}

[1] Y. Liao, H. Sodano, Model of a single mode energy harvester and properties for optimal power generation, Smart Materials and Structures 17 (2008) 065026. 
[2] N. Stephen, On energy harvesting from ambient vibration, Journal of Sound and Vibration 293 (2006) 409-425.

[3] E. Lefeuvre, A. Badel, C. Richard, D. Guyomar, Piezoelectric energy harvesting device optimization by synchronous electric charge extraction, Journal of Intelligent Material Systems and Structures 16 (2005) 865-876.

[4] P. Mitcheson, T. Toh, K. Wong, S. Burrow, A. Holmes, Tuning the resonant frequency and damping of an energy harvester using power electronics, IEEE Transactions on Circuits and Systems 58 (Part 2) (2011) 792-796.

[5] G. Szarka, B. Stark, S. Burrow, Review of power management for kinetic energy harvesting systems, IEEE Transactions on Power Electronics 27 (2012) 803-815.

[6] G. Szarka, S. Burrow, P. Proynov, B. Stark, Maximum power transfer tracking for ultra-low-power electromagnetic energy harvesters, IEEE transactions on Power Electronics 29 (1) (2014) 201-212.

[7] A. Cammarano, S. Burrow, D. Barton, A. Carrella, L. Clare, Tuning a resonant energy harvester using a generalized elelectric load, Smart Materials and Structures 19 (5) (2011) 055003.

[8] N. duToit, B. Wardle, Experimental verification of models for microfabricated piezoelectric vibration energy harvesters, AIAA Journal 45 (5) (2007) 1126-1137.

[9] T. Seuaciuc-Osorio, M. Daqaq, Energy harvesting under excitations of time-varying frequency, Journal of Sound and Vibration 329 (2010) $2497-2515$.

[10] D. Quinn, A. Triplett, L. Bergman, A. Vakakis, Comparing linear and essentially nonlinear vibration-based energy harvesting, Journal of Vibration and Acoustics 133 (2011) 011001-1.

[11] B. Mann, N. Sims, Energy harvesting from the nonlinear oscillations of magnetic levitation, Journal of Sound and Vibration 319 (2009) 515530 . 
[12] A. Triplett, D. Quinn, Experimental investigation of energy harvesting with essential nonlinearities, in: Proceedings of ASME IDETC/CIE, Washington DC., Washington DC.

[13] T. Ma, H. Zhang, Enhancing mechanical energy harvesting with dynamics escaped from potential well, Applied Physics Letters 100 (2012) 114107.

[14] T. Ma, H. Zhang, Reaping the potential of nonlinear energy harvesting with tunable damping and modulation of the forcing functions, Applied Physics Letters 104 (2014) 214104.

[15] A. Erturk, J. Hoffmann, D. Inman, A piezomagnetoelastic structure for broadband vibration energy harvesting, Applied Physics Letters 94 (2009) 254102.

[16] D. Kremer, K. Liu, A nonlinear energy sink with an energy harvester: transient responses, Journal of Sound and Vibration (2014).

[17] Y. Hu, H. Xue, J. Yang, Q. Jiang, Nonlinear behavior of a piezoelectric power harvester near resonance, IEEE Transactions on Ultrasonics, Ferroelectrics and Frequency Control 53 (7) (2006) 1387-1391.

[18] D. Andersen, A. Vakakis, Y. Starosvetsky, L. Bergman, Dynamic instabilities in coupled oscillators induced by geometrically nonlinear damping, Nonlinear Dynamics 67 (2012) 807-827.

[19] A. Vakakis, Inducing passive nonlinear energy sinks in linear vibrating systems, Journal of Vibration and Acoustics 123 (3) (2001) 324-332.

[20] A. Vakakis, L. Manevitch, O. Gendelman, L. Bergman, Dynamics of linear discrete systems connected to local essentially nonlinear attachments, Journal of Sound and Vibration 264 (2003) 559-577.

[21] G. Kerschen, Y. Lee, A. Vakakis, D. McFarland, Irreversible passive energy transfer in coupled oscillators with essential nonlinearity, SIAM Journal on Applied Mathematics 66 (2) (2006) 648-679.

[22] T. Sapsis, D. Quinn, A. Vakakis, L. Bergman, Effective stiffening and damping enhancement of structures with strongly nonlinear local attachments, Journal of Vibration and Acoustics 134 (1) (2012) 0110016. 
[23] D. Andersen, Y. Starosvetsky, M. Mane, S. Hubbard, K. Remick, X. Wang, A. Vakakis, L. Bergman, Non-resonant damped transitions resembling continuous resonance scattering in coupled oscillators with essential nonlinearities, Physica D 241 (2012) 964-974.

[24] A. Itin, A. Neishtadt, A. Vasiliev, Captures into resonance and scattering on resonance in dynamics of a ccharge relativistic particle in magnetic field and electrostatic wave, Physica D 141 (2000) 281-296.

[25] G. Kerschen, O. Gendelman, A. Vakakis, L. Bergman, D. McFarland, Impulsive periodic and quasi-periodic orbits of coupled oscillators with essential stiffness nonlinearity, Communications in Nonlinear Science and Numerical Simulation 13 (2008) 959-978.

[26] K. Remick, A. Vakakis, L. Bergman, D. McFarland, D. Quinn, Sustained high-frequency dynamic instability of a nonlinear system of coupled oscillators forced by single or repeated impulses: theoretical and experimental results, Journal of Vibration and Acoustics 136 (1) (2014) 011013.

[27] K. Remick, H. Joo, D. McFarland, T. Sapsis, L. Bergman, D. Quinn, A. Vakakis, Sustained high-frequency energy harvesting through a strongly nonlinear electromechanical system under single and repeated impulsive excitations, Journal of Sound and Vibration 333 (2014) 32143235 .

[28] S. Beeby, T. O'Donnel, Energy Harvesting Technologies, Springer. New York, New York., 2009.

[29] H. Wheeler, Simple inductance formulas for radio coils, Proceedings of the Institute of Radio Engineers 16 (1928) 1398-1400.

[30] A. Vakakis, O. Gendelman, L. Bergman, D. McFarland, G. Kerschen, Y. Lee, Passive Nonlinear Targeted Energy Transfer in Mechanical and Structural Systems: I and II, Springer Verlag, Berlin and New York., 2008 .

[31] N. Minorsky, Nonlinear oscillations, Van Nostrand, Princeton (1962).

[32] B. D’Urso, R. Van Handel, B. Odom, D. Hanneke, G. Gabrielse, Singleparticle self-excited oscillator, Physical Review Letters 94 (2005) 113002. 
[33] S. Masri, T. Caughey, A nonparametric identification technique for nonlinear dynamic problems, ASME Journal of Applied Mechanics 46 (1979) 433-447.

[34] K. Worden, Data processing and experiment design for the restoring force surface method, part i: Integration and differentiation of measured time data, Mechanical Systems Signal Processing 4(4) (1990) 295-319. 